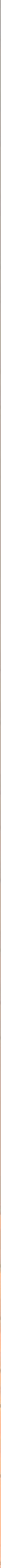




\title{
Actions of some pointed Hopf algebras on path algebras of quivers
}

\author{
Ryan Kinser and Chelsea Walton
}

\begin{abstract}
We classify Hopf actions of Taft algebras $T(n)$ on path algebras of quivers, in the setting where the quiver is loopless, finite, and Schurian. As a corollary, we see that every quiver admitting a faithful $\mathbb{Z}_{n}$-action (by directed graph automorphisms) also admits inner faithful actions of a Taft algebra. Several examples for actions of the Sweedler algebra $T(2)$ and for actions of $T(3)$ are presented in detail. We then extend the results on Taft algebra actions on path algebras to actions of the Frobenius-Lusztig kernel $u_{q}\left(\mathfrak{s l}_{2}\right)$, and to actions of the Drinfeld double of $T(n)$.
\end{abstract}

$\begin{array}{ll}\text { 1. Introduction } & 117 \\ \text { 2. Background } & 121 \\ \text { 3. Preliminary results } & 125 \\ \text { 4. Minimal quivers } & 129 \\ \text { 5. Sweedler algebra actions on path algebras of minimal quivers } & 131 \\ \text { 6. Taft algebra actions on path algebras of minimal quivers } & 135 \\ \text { 7. Gluing Taft algebra actions on minimal quivers } & 136 \\ \text { 8. Extended actions of other pointed Hopf algebras } & 144 \\ \text { 9. Appendix } & 149 \\ \text { Acknowledgments } & 153 \\ \text { References } & 153\end{array}$

\section{Introduction}

Let $n$ be an integer $\geq 2$ and let $\mathbb{k}$ be a field containing a primitive $n$-th root of unity $\zeta$. Both $\mathbb{k}$ and $n$ will be fixed but arbitrary subject to this condition throughout the paper. Note that if $\operatorname{char}(\mathbb{k})=p>0$, this implies that $p$ and $n$ are coprime. All algebras in this work are associative $\mathbb{k}$-algebras and let an unadorned $\otimes$ denote $\otimes_{\mathbb{k}}$.

Generalizing the classical notion of a group acting on an algebra by automorphisms, one can consider actions of Hopf algebras (e.g., quantum groups). However, one obstacle is that the intricate structure of a Hopf algebra often prevents nontrivial actions on an algebra. When such actions exist, they can be difficult to construct and

MSC2010: primary 16T05; secondary 05C20, 16S99.

Keywords: Hopf action, module algebra, path algebra, Schurian quiver, Taft algebra. 
are not generally well understood. This paper presents a case where a classification of these actions is achieved. Here, we consider actions of some finite dimensional, pointed Hopf algebras, namely actions of Taft algebras (Definition 2.1) as a start. Taft algebras can be thought of as Borel subalgebras of the Frobenius-Lusztig kernel $u_{q}\left(\mathfrak{s l}_{2}\right)$. The algebra being acted upon is the path algebra of a quiver, and actions are subject to Hypothesis 1.2. All necessary background, including definitions, is recalled in Section 2. In particular, we address the following question:

Question 1.1. When does the path algebra of a quiver admit a nontrivial action of a (finite dimensional, pointed) Hopf algebra? Specifically, of a Taft algebra?

Actions by Taft algebras are referred to as Taft actions for short. We give a complete answer to the question above for Taft actions, and extend Taft actions to actions of the quantum group $u_{q}\left(\mathfrak{s l}_{2}\right)$ and actions of the Drinfeld double of a Taft algebra, under the following conditions.

Hypothesis 1.2. Unless stated otherwise, we impose the assumptions below:

(a) The quiver $Q$ is finite, loopless, and Schurian.

(b) Hopf actions preserve the ascending filtration by path length of the path algebra $\mathbb{k} Q$.

It is easy to see that $Q$ must at least admit a nontrivial action of the cyclic group $\mathbb{Z}_{n}$ (namely, the group of grouplike elements of $T(n)$ ) to admit a nontrivial action of the $n$-th Taft algebra $T(n)$; see Example 3.13. Since Hopf algebras and quantum groups are generalizations of group algebras, we are interested in when a path algebra of a quiver that admits classical cyclic symmetry admits additional "quantum symmetry", loosely speaking. Our strategy is to identify a class of quivers which is small enough so that we can explicitly describe all Taft actions on their path algebras, but large enough so that every quiver admitting a Taft action is a union of quivers in this class. We call these quivers the $\mathbb{Z}_{n}$-minimal quivers (Definition 4.3). The reader may wish to look over Section 5 early on for a complete account of the case $n=2$ : actions of the Sweedler algebra $T(2)$ on $\mathbb{Z}_{2}$-minimal quivers.

To begin, we first note that any action on a path algebra must restrict to an action on the subalgebra generated by the vertices, by Hypothesis 1.2(b). So we start by classifying Taft actions on products of fields in Proposition 3.5. Then, the form of actions on vertices places significant restrictions on actions on the arrows. The following theorem summarizes our results, with reference to more detailed statements in the body of the paper. Here, we let $g$ and $x$ be the standard generators of $T(n)$, where $g$ is grouplike, $x$ is $(1, g)$ skew-primitive, and $x g=\zeta g x$ for $\zeta$ some primitive $n$-th root of unity (see Section 2A). We identify the cyclic group generated by $g$ with $\mathbb{Z}_{n}$. 
Theorem 1.3. Let $Q$ be a quiver, and suppose we have a Taft action on its path algebra $\mathbb{k} Q$.

(a) The Taft action determines an action of $\mathbb{Z}_{n}$ on $Q$ by quiver automorphisms (Lemma 3.2).

(b) Each $\mathbb{Z}_{n}$-orbit of vertices is stable under $T(n)$. If we let $\left\{e_{1}, \ldots, e_{m}\right\}$ be the collection of trivial paths corresponding to some orbit of vertices, numbered so that $g \cdot e_{i}=e_{i+1}$ with subscripts taken modulo $m$, then the action of $x$ on these is given by

$$
x \cdot e_{i}=\gamma \zeta^{i} e_{i}-\gamma \zeta^{i+1} e_{i+1},
$$

for any scalar $\gamma \in \mathbb{k}$ (Proposition 3.5).

(c) For each arrow a of $Q$, the action of $x$ on a is given by

$$
x \cdot a=\alpha a+\beta(g \cdot a)+\lambda \sigma(a),
$$

for some scalars $\alpha, \beta$, and $\lambda$. Here, $\sigma(a)$ is an arrow or trivial path with the same source as $a$ and the same target as $g \cdot a$ (Notation 3.9, Proposition 3.10). Furthermore, when $Q$ is a $\mathbb{Z}_{n}$-minimal quiver, these scalars are determined explicitly by the formulae (6.2) and (6.4) (Theorems 6.1, 6.3).

With an explicit parametrization of Taft actions on path algebras of $\mathbb{Z}_{n}$-minimal quivers, it remains to show that this is sufficient to parametrize Taft actions on path algebras of quivers subject to Hypothesis 1.2. To do this, we introduce the notion of a $\mathbb{Z}_{n}$-component of a quiver with $\mathbb{Z}_{n}$-action (Definition 7.1). These are the smallest subquivers of $Q$ which have at least one arrow and are guaranteed to be stable under the action of $T(n)$ for any choice of parameters. Moreover, see Definition 7.4 for the notion of a compatible collection of Taft actions.

Theorem 1.4 (Lemmas 7.2, 7.3, Theorem 7.5, Corollary 7.6). Fix an action of $\mathbb{Z}_{n}$ on a quiver $Q$. Then, $Q$ decomposes uniquely into a union of its $\mathbb{Z}_{n}$ components, and any Taft action on $\mathbb{k} Q$ restricts to an action on each component. Moreover, this decomposition gives a bijection between Taft actions on $\mathbb{k} Q$ and compatible collections of Taft actions on the $\mathbb{Z}_{n}$-components of $Q$. In particular, any path algebra of a quiver with a faithful action of $\mathbb{Z}_{n}$ admits an inner faithful action of the $n$-th Taft algebra $T(n)$.

As mentioned above, we extend these results to get actions of other finite dimensional, pointed Hopf actions on path algebras of quivers.

Theorem 1.5 (Theorems 8.10, 8.21, Section 8C). Fix an action of $\mathbb{Z}_{n}$ on a quiver $Q$. Let $q \in \mathbb{k}$ be a $2 n$-th root of unity. Additional restraints on parameters are determined so that the Taft actions on the path algebra of $Q$ produced in Theorems 1.3 and 1.4 extend to an action of the Frobenius-Lusztig kernel $u_{q}\left(\mathfrak{s l}_{2}\right)$ and to an action of the Drinfeld double of $T(n)$. 
As a consequence of the theorem above, we obtain that path algebras of quivers, that admit $\mathbb{Z}_{n}$-symmetry, are algebras in the category of Yetter-Drinfeld modules over $T(n)$ by Majid [1991]; see also [Radford 2012, Exercise 13.1.6]. Hence, motivated by the process of bosonization, or Radford's biproduct construction to produce (potentially new) Hopf algebras (see [Majid 1994; Radford 2012, Theorems $11.6 .7,11.6 .9]$ ), we pose the following question.

Question 1.6. Let $Q$ be a quiver that admits $\mathbb{Z}_{n}$-symmetry. When does the path algebra $\mathbb{k} Q$ admit the structure of a Hopf algebra in the category of Yetter-Drinfeld modules over $T(n)$ ?

1A. Comparisons to other work. A path algebra $\mathbb{k} Q$ is naturally a coalgebra, where the comultiplication of a path is the sum of all splits of the path. There are previous studies on extending the coalgebra structure on $\mathbb{k} Q$ to a graded Hopf algebra, most notably Cibils and Rosso's work [1997; 2002] on Hopf quivers. Here, when $\mathbb{k} Q$ admits the structure of a Hopf algebra, the group of grouplike elements of $\mathbb{k} Q$ consists of the vertex set $Q_{0}$ of $Q$. Moreover, any arrow $a \in Q_{1}$ is a skewprimitive element as $\Delta(a)=s(a) \otimes a+a \otimes t(a)$. One example of their theory is a construction of $T(n)$ from a Hopf quiver, and in this case it has the regular action on the path algebra of this quiver. Our study produces many more examples of Taft actions on path algebras, as our construction allows for nontrivial actions on path algebras of any quiver that admits $\mathbb{Z}_{n}$-symmetry.

Our work also has some intersection with [Gordienko 2015]. On the one hand, Gordienko works in the setting of Taft actions on arbitrary finite dimensional algebras, whereas path algebras of quivers are not always finite dimensional. For example, Gordienko's Theorem 1 classifies Taft algebra actions on products of matrix algebras, while our Proposition 3.5 only classifies Taft algebra actions on products of fields (equivalently, path algebras of arrowless quivers). On the other hand, Gordienko's classification [2015, Theorem 3] is restricted to actions giving $T(n)$-simple module-algebras, whereas we have classified all Taft actions on path algebras (subject to Hypothesis 1.2). With the exception of special parameter values, the path algebras in this work are not simple with respect to the Taft algebra action: one can easily see from our explicit formulas that the Jacobson radical (the ideal generated by the arrows of $Q$ ) is typically a nontrivial two-sided $T(n)$-invariant ideal.

There is an abundance of literature on both the study of quantum symmetry of graphs and group actions on directed graphs from the viewpoint of operator algebras, including [Banica 2005; Banica et al. 2007; Bates et al. 2012; Bichon 2003; Kumjian and Pask 1999]. Connections to our results merit further investigation.

Other works investigating relations between path algebras of quivers and Hopf algebras can be found in [Chen et al. 2004; Huang and Liu 2010; Huang et al. 2010; van Oystaeyen and Zhang 2004; Zhang 2006]. 
Montgomery and Schneider [2001] provide similar results for actions of Taft algebras, and extended actions of $u_{q}\left(\mathfrak{s l}_{2}\right)$ and of $D(T(n))$, on the commutative algebras: $\mathbb{k}(u)$, and $\mathbb{k}[u] /\left(u^{n}-\beta\right)$ with $\beta \in \mathbb{k}$.

\section{Background}

We begin by defining Taft algebras and Hopf algebra actions. We then discuss path algebras of quivers, which will be acted on by Taft algebras throughout this work.

2A. Taft algebras and Hopf algebra actions. Let $H$ be a Hopf algebra with coproduct $\Delta$, counit $\varepsilon$, and antipode $S$. A nonzero element $g \in H$ is grouplike if $\Delta(g)=g \otimes g$, and the set of grouplike elements of $H$ is denoted by $G(H)$. This forces $\varepsilon(g)=1$ and $S(g)=g^{-1}$. An element $x \in H$ is $\left(g, g^{\prime}\right)$-skew-primitive, for grouplike elements $g, g^{\prime}$ of $H$, when $\Delta(x)=g \otimes x+x \otimes g^{\prime}$. In this case, $\varepsilon(x)=0$ and $S(x)=-g^{-1} x g^{-1}$. The following examples of Hopf algebras will be used throughout this work.

Definition 2.1 (Taft algebra $T(n)$, Sweedler algebra $T(2)$ ). The Taft algebra $T(n)$ is a $n^{2}$-dimensional Hopf algebra generated by a grouplike element $g$ and a $(1, g)$ skew-primitive element $x$, subject to relations:

$$
g^{n}=1, \quad x^{n}=0, \quad x g=\zeta g x
$$

for $\zeta$ a primitive $n$-th root of unity. The 4-dimensional Taft algebra $T(2)$ is known as the Sweedler algebra.

Note that $G(T(n))$ is isomorphic to the cyclic group $\mathbb{Z}_{n}$, generated by $g$.

We now recall basic facts about Hopf algebra actions; refer to [Montgomery 1993] for further details. A left $H$-module $M$ has left $H$-action structure map denoted by $\cdot: H \otimes M \rightarrow M$. We use Sweedler notation $\Delta(h)=\sum h_{1} \otimes h_{2}$ for coproducts.

Definition 2.2 ( $H$-action). Given a Hopf algebra $H$ and an algebra $A$, we say that $H$ acts on $A$ (from the left) if, for all $h \in H$ and $p, q \in A$,

(a) $A$ is a left $H$-module;

(b) $h \cdot(p q)=\sum\left(h_{1} \cdot p\right)\left(h_{2} \cdot q\right)$; and

(c) $h \cdot 1_{A}=\varepsilon(h) 1_{A}$.

In this case, we say that $A$ is a left $H$-module algebra. Equivalently, the multiplication map $\mu_{A}: A \otimes A \rightarrow A$ and unit map $\eta_{A}: \mathbb{k} \rightarrow A$ are morphisms of $H$-modules, so $A$ is an algebra in the monoidal category of left $H$-modules.

For the Taft actions in this work, consider the following terminology. 
Definition 2.3 (extending a $G$-action). Given an action of a group $G$ on an algebra $A$, we say that an action of a Hopf algebra $H$ on $A$ extends the $G$-action on $A$ if the restriction of the $H$-action to $G(H)$ agrees with the $G$-action via some isomorphism $G(H) \simeq G$.

In this paper, we are interested in the case where $G=\mathbb{Z}_{n}$ and $H=T(n)$ in the above definition. Moreover, it is useful to restrict to $H$-actions that do not factor through proper quotient Hopf algebras.

Definition 2.4 (inner faithful). A module $M$ over a Hopf algebra $H$ is inner faithful if the action of $H$ on $M$ does not factor through a quotient Hopf algebra of $H$; that is, $I M \neq 0$ for any nonzero Hopf ideal $I \subset H$. A Hopf action of $H$ on an algebra $A$ is inner faithful if $A$ is inner faithful as an $H$-module.

The following lemma is likely known to experts, but does not seem to be readily accessible in the literature, so we provide a proof.

Lemma 2.5. Every nonzero bi-ideal of $T(n)$ contains $x$. Therefore, a Taft action on an algebra $A$ is inner faithful if and only if $x \cdot A \neq 0$.

Proof. Writing $H:=T(n)$, since $H \cong H^{*}$ as Hopf algebras it suffices to prove the dual statement. Namely, since $x$ generates the radical of $H$, the dual approach is to show that every proper sub-bialgebra of $H$ is contained in the coradical of $H$.

Suppose that $A \subseteq H$ is a nonzero Hopf sub-bialgebra of $H$ which is not contained in the coradical of $H$. We will show that $A=H$. Since the coradical $H_{0}$ of $H$ is the span of the grouplike elements $\left\{g^{i} \mid i=0, \ldots, n-1\right\}$, we have that $A$ contains a nonzero element $f=h x^{j}+$ (terms of lower $x$-degree), where $j \geq 1$ and $h \in H_{0}$. Say, $h=\sum_{d=0}^{i} v_{d} g^{d}$, for $v_{d} \in \mathbb{k}$ with $v_{i} \neq 0$. Since $A$ inherits the coproduct from $H$,

$$
\Delta\left(v_{i} g^{i} x^{j}\right)=\sum_{\ell=0}^{j}\left[\begin{array}{l}
j \\
\ell
\end{array}\right]_{\zeta} v_{i} g^{i} x^{j-\ell} \otimes v_{i} g^{j-\ell+i} x^{\ell},
$$

which is in $A \otimes A$. Here, the equality above holds by [Radford 2012, Lemma 7.3.1]. By the maximality of $j$ and $i$ and by taking $\ell=0$ above, we have that $f \in A$ implies $g^{i} x^{j} \in A$. Now applying $\Delta$ to $g^{i} x^{j}$ yields $g^{i} x^{j-\ell} \in A$ for $\ell=0, \ldots, j$. So, we get $g^{i} x \in A$. Likewise, apply $\Delta$ to $g^{i} x$ to conclude that $g^{i}, g^{i+1} \in A$. Since $g$ has finite multiplicative order, $g^{-i} \in A$ as well. Thus, both $g$ and $x$ are in $A$, so $A=H$, as desired.

So the extension of a faithful cyclic group $\left(\mathbb{Z}_{n}\right)$ action on an algebra $A$ to a Taft algebra $(T(n))$ action is inner faithful if and only if $x \cdot A \neq 0$. To study module algebras of $T(n)$, the following standard fact will also be of use. 
Lemma 2.6. For each $T(n)$-action on an algebra $A$, there is a natural action of $T(n)$ on the opposite algebra $\left(A^{\mathrm{op}}, *\right)$, say denoted by $\diamond$, as follows:

$$
g \diamond p=g^{-1} \cdot p \quad \text { and } \quad x \diamond p=g^{-1} x \cdot p .
$$

This gives a bijection between $T(n)$-actions on $A$ and on $A^{\mathrm{op}}$.

Proof. For any Hopf algebra $H$ and algebra $A$, we get that $A$ is an $H$-module algebra if and only if $\left(A^{\mathrm{op}}, *\right)$ is an $H^{\mathrm{cop}}$-module algebra. Here, $H^{\mathrm{cop}}$ is the coopposite algebra of $H$ and $\Delta^{\text {cop }}=\tau \circ \Delta$ with $\tau\left(\sum h_{1} \otimes h_{2}\right)=\sum h_{2} \otimes h_{1}$. Indeed, $h \cdot(p q)=h \cdot(q * p)=\sum\left(h_{2} \cdot q\right) *\left(h_{1} \cdot p\right)=\sum\left(h_{1} \cdot p\right)\left(h_{2} \cdot q\right)$. The map sending $g$ to $g^{-1}$ and $x$ to $g^{-1} x$ gives an isomorphism $T(n) \cong T(n)^{\text {cop }}$ as Hopf algebras, and the result follows.

2B. Path algebras of quivers. A quiver is another name for a directed graph, in the context where the directed graph is used to define an algebra. Here, we review the basic notions and establish notation. More detailed treatments can be found in the texts [Assem et al. 2006; Schiffler 2014]. Formally, a quiver $Q=\left(Q_{0}, Q_{1}, s, t\right)$ consists of a set of vertices $Q_{0}$, a set of arrows $Q_{1}$, and two functions $s, t: Q_{1} \rightarrow Q_{0}$ giving the source and target of each arrow, visualized as

$$
s(a) \stackrel{a}{\longrightarrow} t(a) .
$$

A path $p$ in $Q$ is a sequence of arrows $p=a_{1} a_{2} \cdots a_{\ell}$ for which $t\left(a_{i}\right)=s\left(a_{i+1}\right)$ for $1 \leq i \leq \ell-1$. (Note that we read paths in left-to-right order.) The length of $p$ is the number of arrows $\ell$. There is also a length 0 trivial path $e_{i}$ at each vertex $i \in Q_{0}$, with $s\left(e_{i}\right)=t\left(e_{i}\right)=i$.

A quiver $Q$ has a path algebra $\mathbb{k} Q$ whose basis consists of all paths in $Q$, and multiplication of basis elements is given by composition of paths whenever it is defined, and 0 otherwise. More explicitly, we have the following definition.

Definition 2.8 (path algebra). The path algebra $\mathbb{k} Q$ of a quiver $Q$ is the $\mathbb{k}$-algebra presented by generators from the set $Q_{0} \sqcup Q_{1}$ with the relations

(a) $\sum_{i \in Q_{0}} e_{i}=1$;

(b) $e_{i} e_{j}=\delta_{i j} e_{i}$ for all $e_{i}, e_{j} \in Q_{0}$; and

(c) $a=e_{s(a)} a=a e_{t(a)}$ for all $a \in Q_{1}$.

Condition (a) is due to the assumption that $\left|Q_{0}\right|<\infty$. Further, $e_{i}$ is a primitive orthogonal idempotent in $\mathbb{k} Q$ for all $i$. So, $\mathbb{k} Q$ is an associative algebra with unit, which is finite dimensional if and only if $Q$ has no path of positive length which starts and ends at the same vertex. Notice that $\mathbb{k} Q$ has a natural ascending filtration by path length. Namely, if we let $F_{i}$ be the subspace of $\mathbb{k} Q$ spanned by paths of length at most $i$, then $F_{i} \cdot F_{j} \subseteq F_{i+j}$. 
Definition 2.9 (Schurian). A quiver $Q$ is said to be Schurian if, given any two vertices $i$ and $j$, there is at most one arrow which starts at $i$ and ends at $j$.

Note that the definition above does not exclude oriented 2-cycles.

Definition 2.10 (covering, gluing, $\circledast$ ). A quiver $Q$ is covered by a collection of subquivers $Q^{1}, \ldots, Q^{r}$ if $Q=\bigcup_{i} Q^{i}$. We say $Q$ is obtained by gluing the collection $Q^{1}, \ldots, Q^{r}$ if the collection covers $Q$, and in addition $Q^{i} \cap Q^{j}$ consists entirely of vertices when $i \neq j$; in this case, write

$$
Q=Q^{1} \circledast \cdots \circledast Q^{r} .
$$

Remark 2.11. If a quiver $Q$ is obtained by gluing subquivers $Q^{1}, \ldots, Q^{r}$, then we get that $\mathbb{k} Q$ is the factor of the free product of path algebras $\mathbb{k} Q^{1} * \cdots * \mathbb{k} Q^{r}$ by the ideal generated by $\left\{e_{i, v}-e_{j, v}\right\}$, where for each pair $(i, j)$, the index $v$ varies over the vertices of $Q^{i} \cap Q^{j}$. Here, $e_{\ell, v}$ indicates the trivial path at $v$, for $v \in\left(Q^{\ell}\right)_{0}$.

2C. Group actions on path algebras of quivers. Now we consider group actions on quivers and on their path algebras.

Definition 2.12 (quiver automorphism). Let $Q, Q^{\prime}$ be quivers, and consider two maps of sets $f_{0}: Q_{0} \rightarrow Q_{0}^{\prime}$ and $f_{1}: Q_{1} \rightarrow Q_{1}^{\prime}$.

(1) We say that the pair $f=\left(f_{0}, f_{1}\right)$ is a quiver isomorphism if each of these maps is bijective, and they form a commuting square with the source and target operations. That is, for all $a \in Q_{1}$ we have

$$
\left(f_{0} \circ s\right)(a)=\left(s \circ f_{1}\right)(a) \text { and } \quad\left(f_{0} \circ t\right)(a)=\left(t \circ f_{1}\right)(a) .
$$

(2) We say that the pair $f=\left(f_{0}, f_{1}\right)$ is a quiver automorphism of $Q$ if $f$ satisfies (1) and $Q^{\prime}=Q$.

(3) A group $G$ is said to act on $Q$, or $Q$ is $G$-stable, if $G$ acts on the sets $Q_{0}$ and $Q_{1}$ such that each element of $G$ acts by a quiver automorphism.

Remark 2.13. A quiver automorphism induces an automorphism of its path algebra, but not every automorphism of a path algebra is of this form. See Lemma 3.2.

Remark 2.14. Given a quiver $Q$, we can form the opposite quiver $Q^{\text {op }}$ by interchanging the source and target functions $s$ and $t$. It is clear from the definition of the path algebra that $\mathbb{k}\left(Q^{\mathrm{op}}\right) \cong(\mathbb{k} Q)^{\mathrm{op}}$. Hence, Lemma 2.6 implies there is a bijection between $T(n)$-actions on $\mathbb{k} Q$ and on $\mathbb{k} Q^{\text {op }}$ given by (2.7). See Remark 5.2 for an illustration. 


\section{Preliminary results}

In this section, we present preliminary results on Taft actions on path algebras of loopless, Schurian quivers. We begin by studying actions on vertices, first giving a simple lemma regarding group actions on $\mathbb{k} Q_{0}$ (Lemma 3.1). Then, we extend this result to classifying Taft actions on $\mathbb{k} Q_{0}$ (Proposition 3.5). Preliminary results on Taft actions on path algebras $\mathbb{k} Q$ are provided (Proposition 3.10), along with an example in the case where $Q_{0}$ is fixed by the group of grouplike elements of $T(n)$ (Example 3.13).

The following lemma is elementary, but we provide the details in any case.

Lemma $3.1\left(G\right.$-action on $\left.\mathbb{k} Q_{0}\right)$. Let $G$ be a group, let $Q_{0}$ be a set of vertices, and let $\left\{e_{i}\right\}_{i \in Q_{0}}$ be the corresponding primitive orthogonal idempotents in $\mathbb{k} Q_{0}$. Then, any $G$-action on the set $Q_{0}$ induces an action on the ring $\mathbb{k} Q_{0}$, given by $g \cdot e_{i}=e_{g \cdot i}$ for each $i \in Q_{0}$ and $g \in G$. Moreover, every $G$-action on $\mathbb{k} Q_{0}$ arises in this way.

Proof. The first statement is clear, so suppose for the converse that we have a $G$-action on $\mathbb{k} Q_{0} \simeq \mathbb{k} \times \mathbb{k} \times \cdots \times \mathbb{k}$. To act as a ring automorphism, each element of $G$ must send a complete collection of primitive orthogonal idempotents to another such collection. But in this case, the set $\left\{e_{i}\right\}_{i \in Q_{0}}$ is the unique such collection. So this set must be permuted by $G$, defining an action of $G$ on the set $Q_{0}$.

Now we turn our attention to group actions on arbitrary path algebras of quivers.

Lemma 3.2 ( $G$-action on $\mathbb{k} Q)$. Let $G$ be a group and $Q$ a quiver which is loopless and Schurian, and suppose that $G$ acts by automorphisms of $\mathbb{k} Q$, preserving the ascending filtration by path length. Then, the action of each $g \in G$ on $Q$ is given by

(i) a quiver automorphism $\phi: Q \rightarrow Q$, along with

(ii) a collection of nonzero scalars $\mu_{a} \in \mathbb{k}^{\times}$, indexed by the arrows a of $Q$,

such that $g \cdot a=\mu_{a} \phi(a)$ for each $a \in Q_{1}$. (To be clear, both $\phi$ and the collection $\mu_{a}$ depend on $g$.)

Proof. Since $G$ preserves the path length filtration, it acts by permutations on the vertex set, by Lemma 3.1. Then for each $g \in G$ and $a \in Q_{1}$, we have $g \cdot a=$ $g \cdot\left(e_{s(a)} a\right)=\left(g \cdot e_{s(a)}\right)(g \cdot a)$, showing that $g \cdot a$ lies in the span of arrows starting at $g \cdot e_{s(a)}$. Similarly, we see that $g \cdot a$ lies in the $\mathbb{k}$-span of arrows with target $g \cdot e_{t(a)}$. Since $Q$ is Schurian, this determines a unique arrow $\phi(a)$, with start $s(g \cdot a)=g \cdot e_{s(a)}$ and target $t(g \cdot a)=g \cdot e_{t(a)}$, such that $g \cdot a$ is a scalar times $\phi(a)$. It is immediate from the definition of $\phi$ that $\phi$ is a quiver automorphism.

Convention 3.3. We sometimes use $g \cdot a$ to label an arrow in a diagram, or refer to $g \cdot a$ as an arrow in exposition, in order to avoid introducing the extra notation $\phi$. In these cases, it is understood that one must actually multiply by a scalar to get an arrow on the nose. 
Next, we study the action of skew-primitive elements on arrowless quivers $Q_{0}$, which then leads to Taft actions on the semisimple algebra $\mathbb{k} Q_{0}$. Since the generator $x$ of $T(n)$ is $(1, g)$-skew-primitive, the relation $e_{i}^{2}=e_{i}$ of $\mathbb{k} Q_{0}$ gives us that

$$
x \cdot e_{i}=x \cdot\left(e_{i}^{2}\right)=e_{i}\left(x \cdot e_{i}\right)+\left(x \cdot e_{i}\right)\left(g \cdot e_{i}\right) \in \operatorname{span}_{\mathbb{k}}\left\{e_{i}, g \cdot e_{i}\right\} .
$$

So, to study extensions of a $G$-action on $\mathbb{k} Q_{0}$ to a $T(n)$-action, we can restrict ourselves to a single $G$-orbit of vertices. From here on, we apply the above results to the case where $G=G(T(n))$ is the cyclic group generated by $g \in T(n)$, which we identify with $\mathbb{Z}_{n}$.

Proposition $3.5\left(T(n)\right.$-actions on $\left.\mathbb{k} Q_{0}\right)$. Let $Q_{0}=\{1,2, \ldots, m\}$ be the vertex set of a quiver, where $m$ divides $n$, and $\mathbb{Z}_{n}$ acts on $\mathbb{k} Q_{0}$ by $g \cdot e_{i}=e_{i+1}$. Here, subscripts are always interpreted modulo $m$.

(i) If $m<n$ (so the $\mathbb{Z}_{n}$-action on $Q_{0}$ is not faithful), then $x$ acts on $\mathbb{k} Q_{0}$ by 0 .

(ii) If $m=n$ (so $\mathbb{Z}_{n}$ acts faithfully on $Q_{0}$ ), then the action of $x$ on $\mathbb{k} Q_{0}$ is exactly of the form

$$
x \cdot e_{i}=\gamma \zeta^{i}\left(e_{i}-\zeta e_{i+1}\right) \quad \text { for all } i,
$$

where $\gamma \in \mathbb{k}$ can be any scalar.

In particular, we can extend the action of $\mathbb{Z}_{n}$ on $\mathbb{k} Q_{0}$ to an inner faithful action of $T(n)$ on $\mathbb{k} Q_{0}$ if and only if $m=n$.

Proof. Assume that we have a $T(n)$-action on $\mathbb{k} Q_{0}$ extended from the $\mathbb{Z}_{n}$-action on $Q_{0}$ in Lemma 3.1. By (3.4), we know that $x \cdot e_{i}=\alpha_{i} e_{i}+\beta_{i} e_{i+1}$ for some scalars $\alpha_{i}, \beta_{i} \in \mathbb{k}$. Then, we have

$$
0=x \cdot 1=x \cdot \sum_{i=1}^{m} e_{i}=\sum_{i=1}^{m} \alpha_{i} e_{i}+\beta_{i} e_{i+1}=\sum_{i=1}^{m}\left(\alpha_{i}+\beta_{i-1}\right) e_{i},
$$

which gives $\beta_{i-1}=-\alpha_{i}$. (Here, $\sum_{i=1}^{m} \beta_{i} e_{i+1}=\sum_{i=1}^{m} \beta_{i-1} e_{i}$ by reindexing.) Now the relation $x g=\zeta g x$ applied to $e_{i}$ gives

$$
\alpha_{i+1} e_{i+1}-\alpha_{i+2} e_{i+2}=\zeta\left(\alpha_{i} e_{i+1}-\alpha_{i+1} e_{i+2}\right),
$$

so that $\alpha_{i+1}=\zeta \alpha_{i}$ for all $i$. Setting $\gamma:=\alpha_{1} \zeta^{-1}$ gives $\alpha_{i}=\zeta^{i} \gamma$, so that (3.6) holds whenever a $T(n)$-action exists. We have assumed that $m$ divides $n$, but on the other hand, $x \cdot e_{i}=x \cdot e_{i+m}$ implies that $\gamma \zeta^{i}=\gamma \zeta^{i+m}$. Thus, $\gamma=\gamma \zeta^{m}$. Hence, whenever $m<n=\operatorname{ord}(\zeta)$, we have $\gamma=0$, and $x$ acts by 0 . This establishes (i).

On the other hand, suppose that $m=n$. We will show that Equation (3.6) defines a $T(n)$-action on $\mathbb{k} Q_{0}$ for any $\gamma \in \mathbb{k}$. A simple substitution verifies that $x g \cdot e_{i}=\zeta g x \cdot e_{i}$. The fact that the $x$-action preserves the relations $e_{i} e_{j}=\delta_{i j} e_{i}$ and $\sum_{i=1}^{n} e_{i}=1$ is also easy to check by substitution. The only tedious part is to 
show that $x^{n}$ acts on $\mathbb{k} Q_{0}$ by 0 , which is verified by Lemma 9.10 in the appendix of computations, using symmetric functions. Now, the $T(n)$-action on $\mathbb{k} Q_{0}$ is inner faithful when $\gamma$ is nonzero, by Lemma 2.5. Therefore, (ii) holds.

Now to study Taft actions on path algebras of Schurian quivers in general, we set the following notation.

Notation 3.9 $(\sigma(a))$. Suppose we have a quiver $Q$ and an action of $\mathbb{Z}_{n} \simeq\langle g\rangle \subset T(n)$ on $\mathbb{k} Q$. Given an arrow $a \in Q_{1}$, we know that there exists at most one path of length less than or equal to 1 from $s(a)$ to $t(g \cdot a)$ since $Q$ is Schurian and loopless. Denote this path by $\sigma(a)$ if it exists, and set $\sigma(a)=0$ otherwise.

To be more explicit, consider the following case: when $g$ fixes neither $s(a)$ nor $t(a)$, and $g \cdot t(a) \neq s(a)$, then $\sigma(a)$ is either an arrow or 0 , and can be visualized in the following diagram.

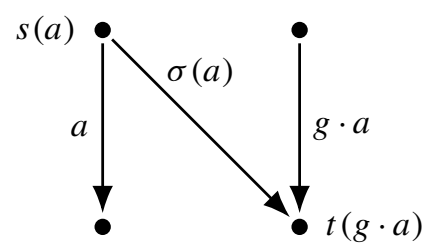

If $g \cdot t(a)=s(a)$, then $\sigma(a)$ is the trivial path at $s(a)$. Moreover, we have $\sigma(a)=g \cdot a$ whenever $g \cdot s(a)=s(a)$, and $\sigma(a)=a$ whenever $g \cdot t(a)=t(a)$.

We remind the reader of the standing assumptions made in Hypothesis 1.2. The following result determines the action of $x$ on any arrow of $Q$.

Proposition 3.10. Suppose we have an action of $T(n)$ on $\mathbb{k} Q$, and let a $\in Q_{1}$ with $i_{+}:=s(a)$ and $j_{-}:=t(a)$. Then, there exist scalars $\alpha, \beta, \lambda \in \mathbb{k}$ such that

$$
x \cdot a=\alpha a+\beta(g \cdot a)+\lambda \sigma(a) .
$$

Moreover, $\alpha, \beta, \lambda$ can be determined in special cases depending on the relative configuration of $a$ and $g \cdot a$, as described in Figures 1 and 2 below. Here, the dotted red arrows indicate the action of $g$ on $Q_{0}$.

Proof. Let $\gamma_{+}, \gamma_{-} \in \mathbb{k}$ be the scalars from Proposition 3.5 such that

$$
x \cdot e_{i_{+}}=\left(\gamma_{+}\right) \zeta^{i}\left(e_{i_{+}}-\zeta g \cdot e_{i_{+}}\right) \text {and } x \cdot e_{j_{-}}=\left(\gamma_{-}\right) \zeta^{j}\left(e_{j_{-}}-\zeta g \cdot e_{j_{-}}\right) \text {, }
$$

where $g \cdot e_{\ell_{ \pm}}=e_{(\ell+1)_{ \pm}}$as usual.

From the relation $a=e_{i_{+}} a$, we can compute

$$
\begin{aligned}
x \cdot a=x \cdot\left(e_{i_{+}} a\right) & =e_{i_{+}}(x \cdot a)+\left(\gamma_{+}\right) \zeta^{i}\left(e_{i_{+}}-\zeta g \cdot e_{i_{+}}\right)(g \cdot a) \\
& = \begin{cases}e_{i_{+}}(x \cdot a)+\left(\gamma_{+}\right) \zeta^{i}(1-\zeta)(g \cdot a) & \text { if } e_{i_{+}}(g \cdot a)=g \cdot a, \\
e_{i_{+}}(x \cdot a)-\left(\gamma_{+}\right) \zeta^{i+1}(g \cdot a) & \text { if } e_{i_{+}}(g \cdot a)=0 .\end{cases}
\end{aligned}
$$




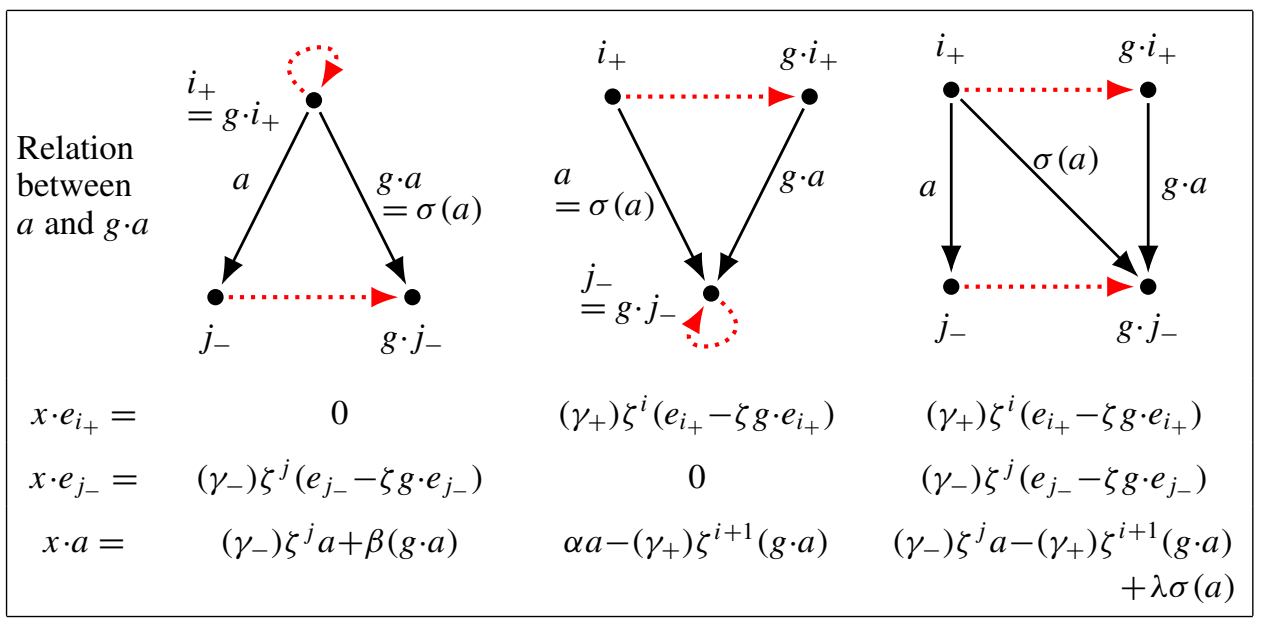

Figure 1. $a(g \cdot a)=(g \cdot a) a=0$.

Thus, $x \cdot a \in \operatorname{span}_{\mathbb{k}}\left\{\right.$ paths starting at $\left.e_{i_{+}}, g \cdot a\right\}$. Similarly, the relation $a=a e_{j_{-}}$gives

$$
\begin{aligned}
x \cdot a=x \cdot\left(a e_{j_{-}}\right) & =\left(\gamma_{-}\right) \zeta^{j} a\left(e_{j_{-}}-\zeta g \cdot e_{j_{-}}\right)+(x \cdot a)\left(g \cdot e_{j_{-}}\right) \\
& = \begin{cases}\left(\gamma_{-}\right) \zeta^{j}(1-\zeta) a+(x \cdot a)\left(g \cdot e_{j_{-}}\right) & \text {if } a\left(g \cdot e_{j_{-}}\right)=a, \\
\left(\gamma_{-}\right) \zeta^{j} a+(x \cdot a)\left(g \cdot e_{j_{-}}\right) & \text {if } a\left(g \cdot e_{j_{-}}\right)=0 .\end{cases}
\end{aligned}
$$

Thus, $x \cdot a \in \operatorname{span}_{\mathbb{k}}\left\{a\right.$, paths ending at $\left.g \cdot e_{j_{-}}\right\}$. Intersecting these two conditions on $x \cdot a$ gives Equation (3.11). The coefficients $\alpha, \beta, \lambda$ can be determined more explicitly, but depend on the relative configuration of $a$ and $g \cdot a$. We consider the various cases below.

Suppose that $e_{i_{+}}(g \cdot a)=g \cdot a$, then $g \cdot s(a)=s(a)$. So, as remarked in Notation 3.9, we have $\sigma(a)=g \cdot a$. We can omit this term in (3.11) by absorbing $\lambda$ into $\beta$ in this case. Similarly, if $a\left(g \cdot e_{j_{-}}\right)=a$, then $g \cdot t(a)=t(a)$ and we have $\sigma(a)=a$. We can omit this term in (3.11) by absorbing $\lambda$ into $\alpha$ in this case. On the other hand,

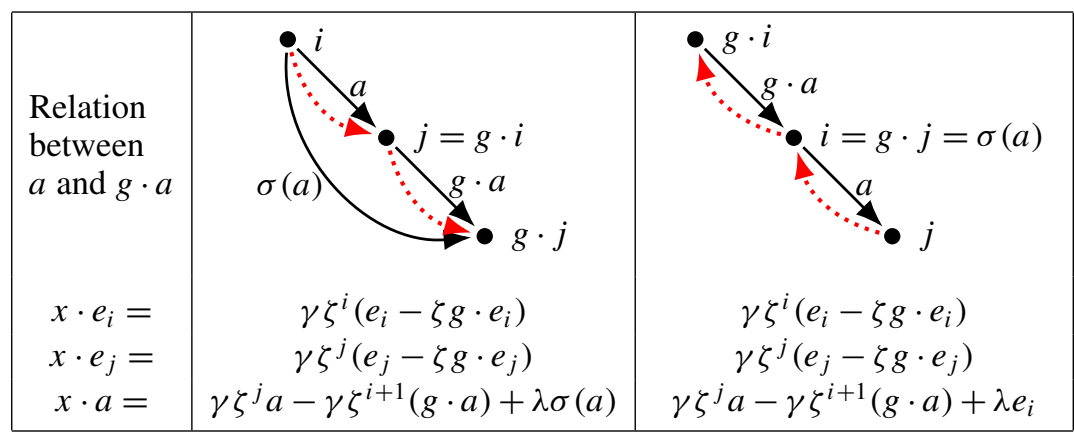

Figure 2. $a(g \cdot a) \neq 0$ or $(g \cdot a) a \neq 0$. 
if $e_{i_{+}}(g \cdot a)=0$, then $x \cdot a=x \cdot e_{i_{+}} a$ implies that

$$
\begin{aligned}
\alpha a+\beta(g \cdot a)+\lambda \sigma(a) & =e_{i_{+}}[\alpha a+\beta(g \cdot a)+\lambda \sigma(a)]-\left(\gamma_{+}\right) \zeta^{i+1}(g \cdot a) \\
& =\alpha a-\left(\gamma_{+}\right) \zeta^{i+1}(g \cdot a)+\lambda \sigma(a) .
\end{aligned}
$$

Thus in this case, $\beta=-\left(\gamma_{+}\right) \zeta^{i+1}$. Similarly, if $a\left(g \cdot e_{j_{-}}\right)=0$, then $x \cdot a=x \cdot a e_{j_{-}}$ implies that

$$
\begin{aligned}
\alpha a+\beta(g \cdot a)+\lambda \sigma(a) & =\left(\gamma_{-}\right) \zeta^{j} a+[\alpha a+\beta(g \cdot a)+\lambda \sigma(a)]\left(g \cdot e_{j_{-}}\right) \\
& =\left(\gamma_{-}\right) \zeta^{j} a+\beta(g \cdot a)+\lambda \sigma(a) .
\end{aligned}
$$

So, $\alpha=\left(\gamma_{-}\right) \zeta^{j}$. These results are collected in Figure 1. In each case, the $x$-action on the vertices follow from Proposition 3.5. In Figure 2, the results are further specialized to the cases where $g \cdot s(a)=t(a)$ or $g \cdot t(a)=s(a)$. Since the \pm notation serves to distinguish the orbits of $s(a)$ and $t(a)$, the \pm notation is dropped in Figure 2.

As an illustration of the results above, we study Taft actions on a path algebra $\mathbb{k} Q$, where $\mathbb{Z}_{n}$ fixes $Q_{0}$.

Example 3.13 $\left(T(n)\right.$-action on $\mathbb{k} Q, \mathbb{Z}_{n}$ fixes $\left.Q_{0}\right)$. If $\mathbb{Z}_{n}$ fixes the vertices of a quiver $Q$, then we claim that any extended action of $T(n)$ on $\mathbb{k} Q$ is not inner faithful. First, by Proposition 3.5(i) with $m=1$, we get that $x \cdot e_{i}=0$ for all $i \in Q_{0}$. For the arrows, we get that $s(g \cdot a)=e_{s(a)}$ and $t(g \cdot a)=e_{t(a)}$ by the assumption. So, $\sigma(a)=g \cdot a$. Moreover, $g \cdot a=\mu_{a} a$ by Lemma 3.2. Now, Proposition 3.10 implies that $x \cdot a=\alpha a$ for some $\alpha \in \mathbb{k}$. Finally, using the relation $x^{n}=0$, we conclude that $x \cdot a=0$, and the claim holds.

\section{Minimal quivers}

Given any Hopf algebra action on an algebra $A$, it is natural to study the restricted Hopf action on a subalgebra of $A$, if one exists. We introduce $\mathbb{Z}_{n}$-minimal quivers in this section, which will be the building blocks of Taft actions on path algebras of quivers in subsequent sections. The following definition serves to fix notation for specific quivers that will be used throughout the rest of the paper.

Definition 4.1 $\left(K_{m}, K_{m, m^{\prime}}, a_{j}^{i}, b_{j}^{i}\right)$. Let $m$ and $m^{\prime}$ be positive integers.

(1) The complete quiver $K_{m}$ (or complete digraph) has vertex set $\{1,2, \ldots, m\}$, with an arrow $a_{j}^{i}$ from $i$ to $j$ for every ordered pair of distinct vertices $(i, j)$. For uniformity in the formulas, we also take the symbol $a_{i}^{i}$ to mean the trivial path $e_{i}$ at vertex $i$ (rather than a loop, which we have excluded).

(2) The complete bipartite quiver $K_{m, m^{\prime}}$ has a top row of $m$ vertices and a bottom row of $m^{\prime}$ vertices, labeled by $\left\{1_{+}, \ldots, m_{+}\right\}$and $\left\{1_{-}, \ldots, m_{-}^{\prime}\right\}$, respectively. 
There is an arrow $b_{j}^{i}$ from each vertex $i_{+}$in the top row to each vertex $j_{-}$in the bottom row; that is, $s\left(b_{j}^{i}\right)=i_{+}$and $t\left(b_{j}^{i}\right)=j_{-}$.

An example of each type is given below, without vertex or arrow labels.
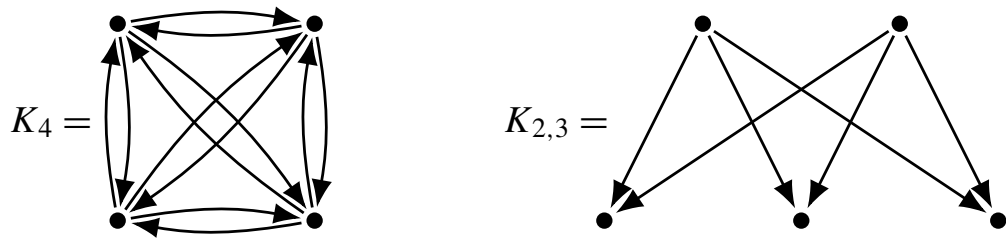

To illustrate the arrow labels, the first diagram below shows some arrows in $K_{4}$ and the second diagram shows all arrows starting at $1^{+}$in $K_{2,3}$.
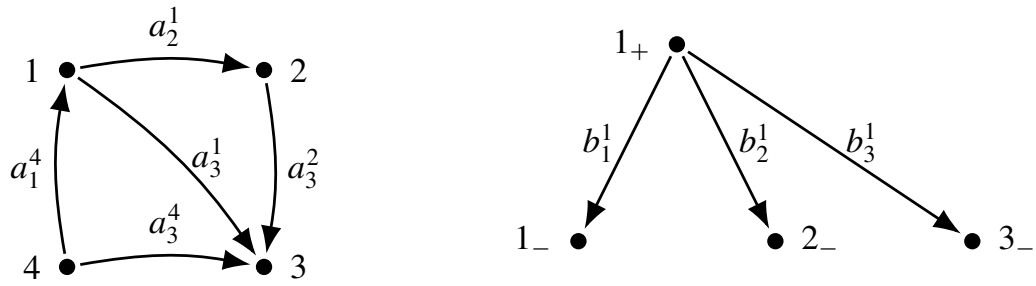

See Figure 5 for further illustrations.

Suppose we have an action of $\mathbb{Z}_{n}$ on the path algebra of a subquiver of $K_{m}$ or $K_{m, m^{\prime}}$. Let $g$ be a generator of $\mathbb{Z}_{n}$. After possibly relabeling, we can assume $g$ acts on the trivial paths by $g \cdot e_{i}=e_{i+1}$ (for $K_{m}$ ) and $g \cdot e_{i_{+}}=e_{(i+1)_{+}}, g \cdot e_{i_{-}=(i+1)_{-}}$(for $K_{m, m^{\prime}}$ ), where the indices are taken modulo $m$ or $m^{\prime}$ as appropriate. By Lemma 3.2, there is a collection of nonzero scalars $\mu_{i, j}$ such that $g \cdot a_{j}^{i}=\mu_{i, j} a_{j+1}^{i+1}$ (for $K_{m}$ ), or $g \cdot b_{j}^{i}=\mu_{i, j} b_{j+1}^{i+1}$ (for $K_{m, m^{\prime}}$ ). Again, subscripts and superscripts are interpreted modulo $m$ or $m^{\prime}$ as appropriate. Since $g^{n}$ is the identity and $g \cdot e_{i}=e_{i+1}$ (for $K_{m}$ ), these scalars $\mu_{i, j}$ satisfy

$$
\begin{aligned}
\mu_{i, i}=1 & \text { in the } K_{m} \text { case, } \\
\prod_{\ell=0}^{n-1} \mu_{i+\ell, j+\ell}=1 & \text { in both cases. }
\end{aligned}
$$

Since an arrow (or, more specifically, the source and target of an arrow) of a quiver $Q$ can only be part of one or two $\mathbb{Z}_{n}$-orbits of $Q_{0}$, we make the following definition.

Definition $4.3\left(\mathbb{Z}_{n}\right.$-minimal, Type A, Type B). Let $\mathbb{Z}_{n}$ act on a quiver $Q$. Say that $Q$ is $\mathbb{Z}_{n}$-minimal of

- Type $A$ if $Q$ is a $\mathbb{Z}_{n}$-stable subquiver of $K_{m}$, where $m>1$ is a positive integer dividing $n$; or 

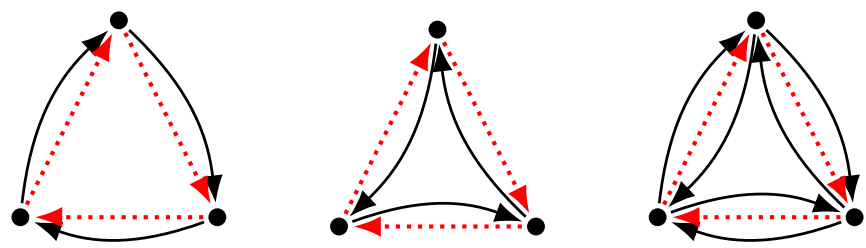

Figure 3. $\mathbb{Z}_{3}$-minimal quivers of Type A.

- Type $B$ if $Q$ is a $\mathbb{Z}_{n}$-stable subquiver of $K_{m, m^{\prime}}$, where $m, m^{\prime} \geq 1$ and $m, m^{\prime}$ divide $n$.

Example 4.4. The following quiver $Q$ admits both a $\mathbb{Z}_{4}$-action and a $\mathbb{Z}_{2}$-action illustrated by the dotted red arrows below. Observe that $Q$ is $\mathbb{Z}_{4}$-minimal of Type B. Further, we see it is $\mathbb{Z}_{4 d}$-minimal of Type B for any integer $d \geq 1$. However, it is not $\mathbb{Z}_{2}$-minimal of Type B.

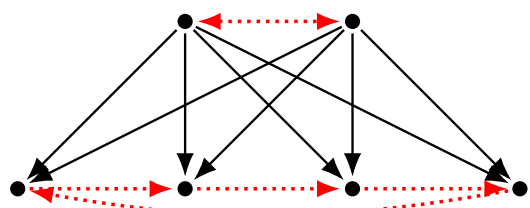

$\mathbb{Z}_{4 d}$-minimal

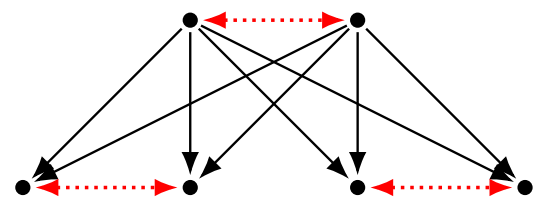

not $\mathbb{Z}_{2}$-minimal

Now we list the $\mathbb{Z}_{n}$-minimal quivers for small $n$. For Type A, note that there is only one $\mathbb{Z}_{2}$-minimal quiver that admits a transitive action of $\mathbb{Z}_{2}$ on vertices; see (I) of Figure 5. See Figure 3 for the three $\mathbb{Z}_{3}$-minimal quivers of Type A. The dotted, red arrow indicates the action of $\mathbb{Z}_{3}$ on $Q_{0}$ (clockwise rotation in each case). For Type B, there are five Type $\mathrm{B} \mathbb{Z}_{2}$-minimal quivers; see Figure $5(\mathrm{II})-(\mathrm{VI})$ for an illustration. Moreover, see Figure 4 for the six $\mathbb{Z}_{3}$-minimal quivers of Type B.

\section{Sweedler algebra actions on path algebras of minimal quivers}

In this section, we study the action of the Sweedler algebra $T(2)$ on path algebras of quivers. This is achieved by first computing the action of the Sweedler algebra on $\mathbb{Z}_{2}$-minimal quivers (Theorem 5.1). Later, in Section 7, we present results on gluing such actions to yield Sweedler actions on more general quivers.

Recall from Definition 2.1 that the Sweedler algebra is the 4-dimensional Taft algebra $T(2)$ generated by a grouplike element $g$ and a $(1, g)$-skew-primitive element $x$, subject to relations:

$$
g^{2}=1, \quad x^{2}=0, \quad x g+g x=0 .
$$

Note that $G(T(2)) \simeq \mathbb{Z}_{2}$. 


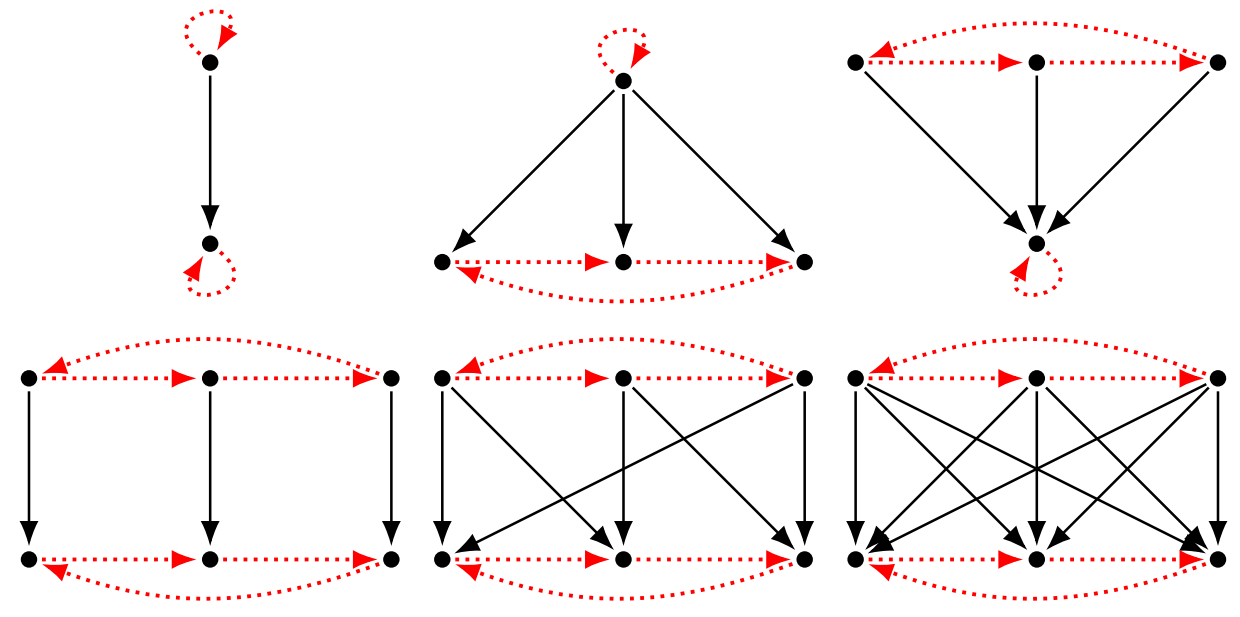

Figure 4. $\mathbb{Z}_{3}$-minimal quivers of Type B.

Theorem 5.1. Recall Hypothesis 1.2. For each quiver $Q$ in Figure 5 (where the dotted red arrow illustrates the g-action on vertices), the $\mathbb{Z}_{2}$-action on $Q$ extends to an action of the Sweedler algebra $T(2)$ on $\mathbb{k} Q$ precisely as follows. We take $\gamma$

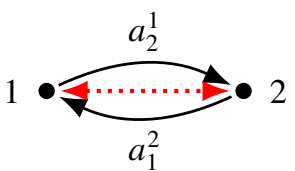

( 1$)$

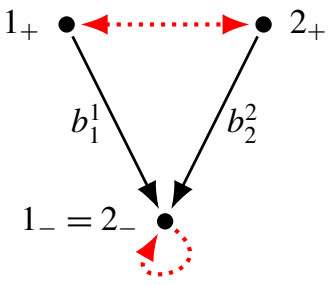

(IV)

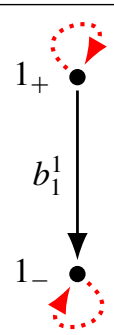

(II)

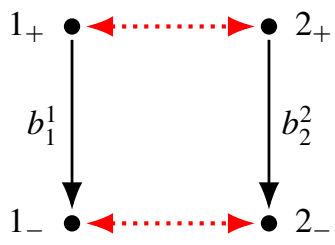

$(\mathrm{V})$

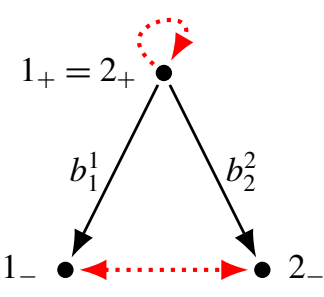

(III)

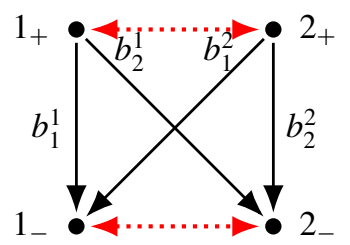

$(\mathrm{VI})$

Figure 5. The $\mathbb{Z}_{2}$-minimal quivers. 
(resp. $\gamma_{+}$and $\gamma_{-}$) to be the scalar from the $x$-action on $e_{i}$ (resp. on $e_{i_{+}}$and on $e_{j_{-}}$) in (3.6) (resp. in (3.12)).

\begin{tabular}{|c|c|c|c|}
\hline$Q$ & $x$-action on $Q_{0}$ & $Q$ & $x$-action on $Q_{0}$ \\
\hline (I) & $\begin{array}{l}x \cdot e_{1}=-\gamma\left(e_{1}+e_{2}\right) \\
x \cdot e_{2}=\gamma\left(e_{1}+e_{2}\right)\end{array}$ & (IV) & $\begin{array}{l}x \cdot e_{1_{+}}=-\left(\gamma_{+}\right)\left(e_{1_{+}}+e_{2_{+}}\right) \\
x \cdot e_{2_{+}}=\left(\gamma_{+}\right)\left(e_{1_{+}}+e_{2_{+}}\right) \\
x \cdot e_{1_{-}}=0\end{array}$ \\
\hline (II) & $x \cdot e_{1_{+}}=x \cdot e_{1_{-}}=0$ & $(\mathrm{~V})$ & $\begin{array}{l}x \cdot e_{1_{+}}=-\left(\gamma_{+}\right)\left(e_{1_{+}}+e_{2_{+}}\right) \\
x \cdot e_{2_{+}}=\left(\gamma_{+}\right)\left(e_{1_{+}}+e_{2_{+}}\right) \\
x \cdot e_{1_{-}}=-\left(\gamma_{-}\right)\left(e_{1_{-}}+e_{2_{-}}\right) \\
x \cdot e_{2_{-}}=\left(\gamma_{-}\right)\left(e_{1_{-}}+e_{2_{-}}\right)\end{array}$ \\
\hline (III) & $\begin{array}{l}x \cdot e_{1_{+}}=0 \\
x \cdot e_{1_{-}}=-\left(\gamma_{-}\right)\left(e_{1_{-}}+e_{2_{-}}\right) \\
x \cdot e_{2_{-}}=\left(\gamma_{-}\right)\left(e_{1_{-}}+e_{2_{-}}\right)\end{array}$ & $(\mathrm{VI})$ & $\begin{array}{l}x \cdot e_{1_{+}}=-\left(\gamma_{+}\right)\left(e_{1_{+}}+e_{2_{+}}\right) \\
x \cdot e_{2_{+}}=\left(\gamma_{+}\right)\left(e_{1_{+}}+e_{2_{+}}\right) \\
x \cdot e_{1_{-}}=-\left(\gamma_{-}\right)\left(e_{1_{-}}+e_{2_{-}}\right) \\
x \cdot e_{2_{-}}=\left(\gamma_{-}\right)\left(e_{1_{-}}+e_{2_{-}}\right)\end{array}$ \\
\hline
\end{tabular}

\begin{tabular}{|c|c|c|}
\hline$Q$ & $x$-action on $Q_{1}$ & \\
\hline (I) & $\begin{array}{l}x \cdot a_{2}^{1}=\gamma a_{2}^{1}-\gamma \mu a_{1}^{2}+\lambda e_{1} \\
x \cdot a_{1}^{2}=\gamma \mu^{-1} a_{2}^{1}-\gamma a_{1}^{2}-\lambda \mu^{-1} e_{2}\end{array}$ & for $\lambda \in \mathbb{k}, \mu \in \mathbb{k}^{\times}$ \\
\hline (II) & $x \cdot b_{1}^{1}=0$ & \\
\hline (III) & $\begin{array}{l}x \cdot b_{1}^{1}=-\left(\gamma_{-}\right) b_{1}^{1}+\beta \mu b_{2}^{2} \\
x \cdot b_{2}^{2}=-\beta \mu^{-1} b_{1}^{1}+\left(\gamma_{-}\right) b_{2}^{2}\end{array}$ & for $\beta^{2}=\left(\gamma_{-}\right)^{2}, \mu \in \mathbb{k}^{\times}$ \\
\hline (IV) & $\begin{array}{l}x \cdot b_{1}^{1}=\alpha b_{1}^{1}-\left(\gamma_{+}\right) \mu b_{2}^{2} \\
x \cdot b_{2}^{2}=\left(\gamma_{+}\right) \mu^{-1} b_{1}^{1}-\alpha b_{2}^{2}\end{array}$ & for $\alpha^{2}=\left(\gamma_{+}\right)^{2}, \mu \in \mathbb{k}^{\times}$ \\
\hline$(\mathrm{V})$ & $\begin{array}{l}x \cdot b_{1}^{1}=-\left(\gamma_{-}\right) b_{1}^{1}-\left(\gamma_{+}\right) \mu b_{2}^{2} \\
x \cdot b_{2}^{2}=\left(\gamma_{+}\right) \mu^{-1} b_{1}^{1}+\left(\gamma_{-}\right) b_{2}^{2}\end{array}$ & for $\left(\gamma_{+}\right)^{2}=\left(\gamma_{-}\right)^{2}, \mu \in \mathbb{k}^{\times}$ \\
\hline$(\mathrm{VI})$ & $\begin{array}{l}x \cdot b_{1}^{1}=-\left(\gamma_{-}\right) b_{1}^{1}-\left(\gamma_{+}\right) \mu b_{2}^{2}+\lambda b_{2}^{1} \\
x \cdot b_{2}^{2}=\left(\gamma_{+}\right) \mu^{-1} b_{1}^{1}+\left(\gamma_{-}\right) b_{2}^{2}-\lambda \mu^{-1} \mu^{\prime} b_{1}^{2} \\
x \cdot b_{2}^{1}=\left(\gamma_{-}\right) b_{2}^{1}-\left(\gamma_{+}\right) \mu^{\prime} b_{1}^{2}+\lambda^{\prime} b_{1}^{1} \\
x \cdot b_{1}^{2}=\left(\gamma_{+}\right) \mu^{\prime-1} b_{2}^{1}-\left(\gamma_{-}\right) b_{1}^{2}-\lambda^{\prime} \mu \mu^{\prime-1} b_{2}^{2}\end{array}$ & $\begin{array}{l}\text { for }\left(\gamma_{+}\right)^{2}=\left(\gamma_{-}\right)^{2}+\lambda \lambda^{\prime} \\
\text { with } \lambda, \lambda^{\prime} \in \mathbb{k} \\
\text { and } \mu, \mu^{\prime} \in \mathbb{k}^{\times}\end{array}$ \\
\hline
\end{tabular}

Proof. The $x$-action on vertices follows from (3.6) and (3.12).

(I) We are in the situation illustrated in the second column of Figure 2, where additionally $g \cdot i=j$. So, we have

$$
x \cdot a_{2}^{1}=\gamma a_{2}^{1}-\gamma \mu_{1,2} a_{1}^{2}+\lambda e_{1}
$$


from Proposition 3.10 and Lemma 3.2. Then using the relation $x g=-g x$ in $T(2)$, we find that

$$
\mu_{1,2} x \cdot a_{1}^{2}=x \cdot\left(g \cdot a_{2}^{1}\right)=-g \cdot\left(x \cdot a_{2}^{1}\right)=-\gamma \mu_{1,2} a_{1}^{2}+\gamma \mu_{1,2} \mu_{2,1} a_{2}^{1}-\lambda \mu_{1,1} e_{2} .
$$

Using that $\mu_{1,2}^{-1}=\mu_{2,1}$ and $\mu_{1,1}=1$ from Equation (4.2), we obtain

$$
x \cdot a_{1}^{2}=\gamma \mu_{2,1} a_{2}^{1}-\gamma a_{1}^{2}-\lambda \mu_{2,1} e_{2} .
$$

The $x$-action on all other relations in $\mathbb{k} Q$ yields tautologies, and the relations $x^{2}=x g+g x=0$ are satisfied when applied to the arrows $a_{2}^{1}$ and $a_{1}^{2}$. Taking $\mu=\mu_{1,2}$ and $\mu^{-1}=\mu_{2,1}$, we see the action is of the claimed form.

(II) We apply Example 3.13.

(III) Examining Figure 1, we see that $x \cdot b_{1}^{1}=-\left(\gamma_{-}\right) b_{1}^{1}+\beta \mu_{1,1} b_{2}^{2}$ for some $\beta \in \mathbb{k}$. The relation $x g=-g x$ in $T(2)$ then gives

$$
\mu_{1,1} x \cdot b_{2}^{2}=x \cdot\left(g \cdot b_{1}^{1}\right)=-g \cdot\left(x \cdot b_{1}^{1}\right)=-\beta \mu_{1,1} \mu_{2,2} b_{1}^{1}+\left(\gamma_{-}\right) \mu_{1,1} b_{2}^{2} .
$$

Now applying the relation $x^{2}=0$ of $T(2)$ to the arrow $b_{1}^{1}$ implies that $\left(\gamma_{-}\right)^{2}=\beta^{2}$, and we take $\mu=\mu_{1,1}$.

(IV) We get from Figure 1 that $x \cdot b_{1}^{1}=\alpha b_{1}^{1}-\left(\gamma_{+}\right) \mu_{1,1} b_{2}^{2}$ for some $\alpha \in \mathbb{k}$. The relation $x g=-g x$ in $T(2)$ then gives

$$
\mu_{1,1} x \cdot b_{2}^{2}=x \cdot\left(g \cdot b_{1}^{1}\right)=-g \cdot\left(x \cdot b_{1}^{1}\right)=-\alpha \mu_{1,1} b_{2}^{2}+\left(\gamma_{+}\right) \mu_{1,1} \mu_{2,2} b_{1}^{1} .
$$

Now applying the relation $x^{2}=0$ to the arrow $b_{1}^{1}$ implies that $\left(\gamma_{+}\right)^{2}=\alpha^{2}$, and again we take $\mu=\mu_{1,1}$.

(V) Examining Figure 1 we find that $x \cdot b_{1}^{1}=-\left(\gamma_{-}\right) b_{1}^{1}-\left(\gamma_{+}\right) \mu_{1,1} b_{2}^{2}$. Then the relation $x g=-g x$ in $T(2)$ gives that $\mu_{1,1} x \cdot b_{2}^{2}=\left(\gamma_{+}\right) \mu_{1,1} \mu_{2,2} b_{1}^{1}+\left(\gamma_{-}\right) \mu_{1,1} b_{2}^{2}$. The relation $x^{2}=0$ applied to the arrows $b_{1}^{1}$ and $b_{2}^{2}$ implies that $\left(\gamma_{+}\right)^{2}=\left(\gamma_{-}\right)^{2}$, and again we take $\mu=\mu_{1,1}$.

$(\mathrm{VI})$ We can see from Figure 1 that $x \cdot b_{1}^{1}=-\left(\gamma_{-}\right) b_{1}^{1}-\left(\gamma_{+}\right) \mu_{1,1} b_{2}^{2}+\lambda b_{2}^{1}$ for some $\lambda \in \mathbb{R}$. Similarly, we can see from this figure that $x \cdot b_{2}^{1}=\left(\gamma_{-}\right) b_{2}^{1}-\left(\gamma_{+}\right) \mu_{1,2} b_{1}^{2}+\lambda^{\prime} b_{1}^{1}$ for some $\lambda^{\prime} \in \mathbb{k}$. The relation $x g=-g x$ gives the formulas for $x \cdot b_{2}^{2}$ and $x \cdot b_{1}^{2}$ as claimed. Finally, the relation $x^{2}=0$ implies that $\left(\gamma_{+}\right)^{2}=\left(\gamma_{-}\right)^{2}+\lambda \lambda^{\prime}$. We have taken $\mu=\mu_{1,1}$ and $\mu^{\prime}=\mu_{1,2}$.

Remark 5.2. Since $Q(\mathrm{III})^{\mathrm{op}}=Q(\mathrm{IV})$, we can get the Sweedler action on the path algebra of $Q(\mathrm{IV})$ from its action on the path algebra of $Q(\mathrm{III})$ using (2.7); see Remark 2.14. For instance, take $b_{1}^{1} \in Q(\mathrm{IV})$ :

$$
x \diamond b_{1}^{1}=g^{-1} x \cdot b_{1}^{1}=g^{-1} \cdot\left(-\left(\gamma_{-}\right) b_{1}^{1}+\beta \mu b_{2}^{2}\right)=-\left(\gamma_{-}\right) \mu b_{2}^{2}+\beta b_{1}^{1} .
$$

Now by identifying $\gamma_{-}$with $\gamma_{+}$, and $\beta$ with $\alpha$, we get the desired action. 


\section{Taft algebra actions on path algebras of minimal quivers}

In this section, we give a complete description of $T(n)$-actions on path algebras of $\mathbb{Z}_{n}$-minimal quivers. Since the action of the grouplike elements of $T(n)$ has already been determined in Lemma 3.2, and the $T(n)$-action on the vertices has already been determined in Proposition 3.5, all that remains is to describe possible actions of $x$ on the arrows.

6A. Taft actions on Type A $\mathbb{Z}_{n}$-minimal quivers. Let $Q$ be a $\mathbb{Z}_{n}$-minimal quiver of Type A, so that $Q$ is a subquiver of $K_{m}$ for some $m \mid n$ by Definition 4.3. Recall that the path algebra $\mathbb{k} K_{m}$ has basis $a_{j}^{i}$ for $1 \leq i, j \leq m$, with $a_{i}^{i}=e_{i}$ being the trivial path at vertex $i$.

Theorem 6.1. Recall Hypothesis 1.2 and retain the notation above. Any $T(n)-$ action on the path algebra of Type $A \mathbb{Z}_{n}$-minimal quiver $Q$ is given by

$$
x \cdot a_{j}^{i}=\gamma\left(\zeta^{j} a_{j}^{i}-\zeta^{i+1} \mu_{i, j} a_{j+1}^{i+1}\right)+\lambda_{i, j} a_{j+1}^{i},
$$

where $\gamma \in \mathbb{k}$ from (3.6), $\mu_{i, j} \in \mathbb{k}^{\times}$from (4.2), and $\lambda_{i, j} \in \mathbb{k}$ are all scalars satisfying

- $\mu_{i, i}=1$ for all $i$, and $\prod_{\ell=0}^{n-1} \mu_{i+\ell, j+\ell}=1$;

- $\lambda_{i, j}=0$ if either $i=j$ or the arrow $a_{j+1}^{i}$ does not exist in $Q ;$ and

- $\lambda_{i+1, j+1} \mu_{i, j}=\zeta \lambda_{i, j} \mu_{i, j+1}$.

The superindices and subindices of arrows and scalars are taken modulo $\mathrm{m}$.

Proof. We have already used the relations of the path algebra to find that

$$
x \cdot a_{j}^{i}=\gamma\left(\zeta^{j} a_{j}^{i}-\zeta^{i+1} \mu_{i, j} a_{j+1}^{i+1}\right)+\lambda_{i, j} a_{j+1}^{i},
$$

for some scalars $\mu_{i, j} \in \mathbb{k}^{\times}$and $\lambda_{i, j} \in \mathbb{k}$; see Lemma 3.2 and Propositions 3.5 and 3.10. Namely, the case of $i=j$ is Proposition 3.5, which gives $\lambda_{i, i}=0$ and $\mu_{i, i}=1$ for all $i$. Moreover, the case of $i \neq j$ is Proposition 3.10, which gives that $\lambda_{i, j}=0$ if the arrow $a_{j+1}^{i}$ does not exist in $Q$. The condition on the $\left\{\mu_{i, j}\right\}$ is from (4.2).

The relation $x g=\zeta g x$ of $T(n)$ applied to $a_{j}^{i}$ gives on the one hand that

$$
\begin{aligned}
x g \cdot a_{j}^{i} & =x \cdot \mu_{i, j} a_{j+1}^{i+1} \\
& =\gamma \mu_{i, j}\left(\zeta^{j+1} a_{j+1}^{i+1}-\zeta^{i+2} \mu_{i+1, j+1} a_{j+2}^{i+2}\right)+\lambda_{i+1, j+1} \mu_{i, j} a_{j+2}^{i+1},
\end{aligned}
$$

while on the other hand that

$$
\zeta g x \cdot a_{j}^{i}=\zeta \gamma\left(\zeta^{j} \mu_{i, j} a_{j+1}^{i+1}-\zeta^{i+1} \mu_{i, j} \mu_{i+1, j+1} a_{j+2}^{i+2}\right)+\zeta \lambda_{i, j} \mu_{i, j+1} a_{j+2}^{i+1} .
$$

Thus we see that $\lambda_{i+1, j+1} \mu_{i, j}=\zeta \lambda_{i, j} \mu_{i, j+1}$, which is the third condition on the scalars. We also obtain that the relation $x^{n}=0$ applied to $a_{j}^{i}$ imposes no further restrictions on the $x$-action on $a_{j}^{i}$; this is verified in Lemma 9.11 of the appendix. 
6B. Taft actions on Type $B \mathbb{Z}_{n}$-minimal quivers. In this subsection, let $Q$ be a $\mathbb{Z}_{n}$-minimal quiver of Type B. By Definition 4.3, we know that $Q$ is a subquiver of $K_{m, m^{\prime}}$ for some positive integers $m, m^{\prime}$ both dividing $n$. Recall that the path algebra $K_{m, m^{\prime}}$ has basis $e_{i_{+}}, e_{j_{-}}, b_{j}^{i}$ where $1 \leq i \leq m$ and $1 \leq j \leq m^{\prime}$.

Theorem 6.3. Recall Hypothesis 1.2 and retain the notation above. Any T(n)action on the path algebra of Type $B \mathbb{Z}_{n}$-minimal quiver $Q$ is given by

$$
x \cdot b_{j}^{i}=\left(\gamma_{-}\right) \zeta^{j} b_{j}^{i}-\left(\gamma_{+}\right) \mu_{i, j} \zeta^{i+1} b_{j+1}^{i+1}+\lambda_{i, j} b_{j+1}^{i},
$$

where $\gamma_{+}, \gamma_{-} \in \mathbb{k}$ from (3.12), $\mu_{i, j} \in \mathbb{k}^{\times}$from (4.2), and $\lambda_{i, j} \in \mathbb{k}$ are all scalars satisfying

- $\prod_{\ell=0}^{n-1} \mu_{i+\ell, j+\ell}=1$;

- $\lambda_{i, j}=0$ if the arrow $b_{j+1}^{i}$ does not exist in $Q$;

- $\lambda_{i+1, j+1} \mu_{i, j}=\zeta \lambda_{i, j} \mu_{i, j+1}$;

- $\left(\gamma_{+}\right)^{n}=\left(\gamma_{-}\right)^{n}+\prod_{\ell=0}^{n-1} \lambda_{i, j+\ell}$.

The superindices of arrows are taken modulo $m$ and the subindices of arrows are taken modulo $\mathrm{m}^{\prime}$.

Proof. The formula (6.4) and first three conditions on the scalars are derived exactly as in the proof of Theorem 6.1, replacing $a_{\star}^{\star}$ with $b_{\star}^{\star}$ and $\gamma$ with $\gamma_{ \pm}$, appropriately. It just remains to check that $x^{n}$ acts by 0 ; this is equivalent to the last condition on the scalars, as shown in Lemma 9.12.

\section{Gluing Taft algebra actions on minimal quivers}

7A. Gluing actions from components. In this section, we provide a recipe for gluing actions of Taft algebras on minimal quivers. We also show that, given any quiver with $\mathbb{Z}_{n}$-symmetry, one can construct an inner faithful extended action of $T(n)$ on the path algebra of this quiver.

Definition 7.1 ( $\mathbb{Z}_{n}$-component). Let $Q$ be a quiver with an action of $\mathbb{Z}_{n}$, and consider the set of $\mathbb{Z}_{n}$-minimal subquivers of $Q$, partially ordered by inclusion. We say that a $\mathbb{Z}_{n}$-minimal subquiver of $Q$ is a $\mathbb{Z}_{n}$-component of $Q$ if it is maximal in the given ordering.

Lemma 7.2. Fix an action of $\mathbb{Z}_{n}$ on a quiver $Q$. Then, there exists a collection of $\mathbb{Z}_{n}$-components of $Q$, unique up to relabeling, such that $Q$ is obtained by gluing this collection.

Proof. The $\mathbb{Z}_{n}$-components of $Q$ exist and are uniquely determined by the definition because they are the maximal elements of a finite poset. Each arrow of $Q$ lies in some $\mathbb{Z}_{n}$-minimal subquiver, so the $\mathbb{Z}_{n}$-components cover $Q$. So, it suffices to show 
that the intersection of two distinct $\mathbb{Z}_{n}$-components consists entirely of vertices. If $Q^{1}$ and $Q^{2}$ are two $\mathbb{Z}_{n}$-minimal subquivers such that $Q^{1} \cap Q^{2}$ contains an arrow, then we can see from the definition of minimality that $Q^{1}$ and $Q^{2}$ have the same set of vertices. Thus, $Q^{1} \cup Q^{2}$ is a $\mathbb{Z}_{n}$-minimal subquiver of $Q$. Repeat this process to conclude that, by maximality, any two distinct $\mathbb{Z}_{n}$-components can only have vertices in their intersection.

A visualization of the result above can be found in Step 1 of Examples 7.8 and 7.9 below.

Lemma 7.3. Any $T(n)$-action on a path algebra $\mathbb{k} Q$ restricts to an action on the path algebra of each $\mathbb{Z}_{n}$-component of $Q$.

Proof. Let $Q^{i}$ be a $\mathbb{Z}_{n}$-component of $Q$. Since $Q^{i}$ is $\mathbb{Z}_{n}$-minimal, by definition $\mathbb{k} Q^{i}$ is stable under the action of $g$, so it suffices to show that $\mathbb{k} Q^{i}$ is stable under the action of $x$. From Proposition 3.10, it is enough to see that $\sigma(a) \in Q^{i}$ when $a \in Q^{i}$. Suppose not, that is, $\sigma(a) \in Q^{j}$ for some $j \neq i$. Then, $Q^{i} \cup Q^{j}$ is a $\mathbb{Z}_{n}$-minimal quiver, which contradicts the maximality of the $\mathbb{Z}_{n}$-component $Q^{i}$.

Definition 7.4 (compatibility). Let $Q$ be a quiver and $Q^{1}, \ldots, Q^{r} \subseteq Q$ a collection of subquivers of $Q$. Suppose that we have a $T(n)$-action on each path algebra $\mathbb{k} Q^{i}$. We say that this collection of $T(n)$-actions is compatible if, for each pair $(i, j)$, the restriction of the actions on $\mathbb{k} Q^{i}$ and on $\mathbb{k} Q^{j}$ to $\mathbb{k}\left[Q^{i} \cap Q^{j}\right]$ are the same.

Now we have our main result of the section.

Theorem 7.5. Let $Q$ be a quiver with $\mathbb{Z}_{n}$-action. The $T(n)$-actions on the path algebra of $Q$ extending the given $\mathbb{Z}_{n}$-action are in bijection with compatible collections of $T(n)$-actions on path algebras of the $\mathbb{Z}_{n}$-components of $Q$.

Proof. Given a $T(n)$-action on $\mathbb{k} Q$, it restricts to a $T(n)$-action on each path algebra of a $\mathbb{Z}_{n}$-component of $Q$ by Lemma 7.3. On the other hand, suppose we have a collection of compatible $T(n)$-actions on the path algebras of the $\mathbb{Z}_{n}$-components of $Q$. This uniquely determines an action of $T(n)$ on $\mathbb{k} Q$ as follows:

- The action on each arrow of $\mathbb{k} Q$ is uniquely determined since each arrow lies in a unique $\mathbb{Z}_{n}$-component of $Q$.

- The action on any vertex is determined because every vertex lies in at least one $\mathbb{Z}_{n}$-component.

- Suppose that a vertex lies in multiple $\mathbb{Z}_{n}$-components. Then, the action on this vertex is uniquely determined because the actions on different $\mathbb{Z}_{n}$-components restrict to the same action on vertices in their intersection, due to compatibility.

Corollary 7.6. If a quiver $Q$ admits a faithful $\mathbb{Z}_{n}$-action, then $\mathbb{k}_{k} Q$ admits an inner faithful action of the Taft algebra $T(n)$, extending the given $\mathbb{Z}_{n}$-action on $Q$. 
Proof. This follows immediately from Theorem 7.5 and Proposition 3.5 since $Q$ admits an orbit of vertices of size $n$ in this case.

Example 7.7. This example shows that a faithful $\mathbb{Z}_{n}$-action on $Q$ is not necessary for $\mathbb{k} Q$ to admit an inner faithful action of $T(n)$. Fix $\zeta$, a primitive fourth root of unity. Consider the action of $T(4)$ on $\mathbb{k} K_{2}$ (see (I) of Figure 5) given by

$$
g \cdot e_{i}=e_{i+1}, \quad g \cdot a_{j}^{i}=\zeta a_{j+1}^{i+1}, \quad x \cdot e_{i}=0, \quad x \cdot a_{j}^{i}=\lambda e_{i},
$$

for $i \neq j$, where subscripts and superscripts are taken modulo 2 , and $\lambda \in \mathbb{k}^{\times}$is an arbitrary nonzero scalar. By Theorem 6.1, this defines an action of $T(4)$ on the path algebra $\mathbb{k} K_{2}$, which is inner faithful even though the induced action of $\mathbb{Z}_{4}$ on the quiver $K_{2}$ is not faithful.

7B. Algorithm to explicitly parametrize $T(n)$-actions on $\mathbb{k} Q$. Let $Q$ be a quiver that admits an action of $\mathbb{Z}_{n}$. We construct all actions of $T(n)$ on $\mathbb{k} Q$ which extend the given $\mathbb{Z}_{n}$-action via the following steps. Those for which $x$ does not act by 0 are inner faithful by Lemma 2.5. The reader may wish to refer to one of the examples in the next subsection for an illustration of the algorithm below.

First, we know that $Q$ decomposes uniquely into the union of certain $\mathbb{Z}_{n}$-minimal quivers $\left\{Q^{\ell}\right\}_{\ell=1}^{r}$ (namely, $\mathbb{Z}_{n}$-components) so that $Q=Q^{1} \circledast \cdots \circledast Q^{r}$, due to Lemma 7.2.

\section{$\underline{\text { STEP } 1}$}

Decompose $Q$ into this unique union of $\mathbb{Z}_{n}$-components $\left\{Q^{\ell}\right\}$.

Next, we define the extended action of $T(n)$ on the path algebra of each component $Q^{\ell}$. Let $m, m^{\prime}$ be positive divisors of $n$. Recall that Type A and Type B $\mathbb{Z}_{n}$-minimal quivers are subquivers of $K_{m}$ with $m>1$ and of $K_{m, m^{\prime}}$, respectively.

\section{$\underline{\text { STEP } 2}$}

For each $\mathbb{Z}_{n}$-component $Q^{\ell}$ of Type A, label its vertices by $\left\{1^{(\ell)}, \ldots, m^{(\ell)}\right\}$. For each $\mathbb{Z}_{n}$-component $Q^{\ell}$ of Type B, label its sources and sinks by $\left\{1_{+}^{(\ell)}, \ldots, m_{+}^{(\ell)}\right\}$ and $\left\{1_{-}^{(\ell)}, \ldots\left(m^{\prime}\right)_{-}^{(\ell)}\right\}$, respectively.

Recall that $\zeta$ is the primitive $n$-th root of unity from the definition of $T(n)$. Now invoke Proposition 3.5 in the following step.

\section{STEP 3}

Take scalars $\gamma^{(\ell)}, \gamma_{+}^{(\ell)}, \gamma_{-}^{(\ell)} \in \mathbb{k}$ and define

$$
\begin{array}{ll}
x \cdot e_{i^{(\ell)}}=\gamma^{(\ell)} \zeta^{i}\left(e_{i^{(\ell)}}-\zeta e_{\left.(i+1)^{(\ell)}\right)}\right. & \text { for Type A, } \\
x \cdot e_{i_{+}^{(\ell)}}=\gamma_{+}^{(\ell)} \zeta^{i}\left(e_{i_{+}^{(\ell)}}-\zeta e_{(i+1)_{+}^{(\ell)}}\right) & \text { for Type B, } \\
x \cdot e_{i_{-}^{(\ell)}}=\gamma_{-}^{(\ell)} \zeta^{i}\left(e_{i_{-}^{(\ell)}}-\zeta e_{(i+1)_{-}^{(\ell)}}\right) & \text { for Type B, }
\end{array}
$$


where the indices are taken modulo $m$ for Type A, and are taken modulo $m$ or $m^{\prime}$ for Type B. Here, $\gamma^{(\ell)}=0$ if $m<n$ for Type A and $\gamma_{+}^{(\ell)}=0$ or $\gamma_{-}^{(\ell)}=0$ if $m<n$ or $m^{\prime}<n$ respectively, for Type B. To obtain compatibility, impose relations amongst the scalars by identifying vertices.

Finally, we invoke Theorems 6.1 and 6.3 to get all actions of $T(n)$ on the arrows of $Q$.

\section{STEP 4}

Label the arrows of each Type A and Type B component of $Q$ by arrows $\left(a_{j}^{i}\right)^{(\ell)}$ and $\left(b_{j}^{i}\right)^{(\ell)}$ respectively.

\section{$\underline{\text { STEP } 5}$}

Given the scalars $\gamma^{(\ell)}, \gamma_{+}^{(\ell)}, \gamma_{-}^{(\ell)} \in \mathbb{k}$ of Step 3, we have, for all arrows $a_{j}^{i}$ of a component of Type A and $b_{j}^{i}$ of a component of Type $\mathrm{B}$, that

$$
\begin{aligned}
& x \cdot\left(a_{j}^{i}\right)^{(\ell)}=\gamma^{(\ell)}\left(\zeta^{j}\left(a_{j}^{i}\right)^{(\ell)}-\zeta^{i+1} \mu_{i, j}^{(\ell)}\left(a_{j+1}^{i+1}\right)^{(\ell)}\right)+\lambda_{i, j}^{(\ell)}\left(a_{j+1}^{i}\right)^{(\ell)}, \\
& x \cdot\left(b_{j}^{i}\right)^{(\ell)}=\gamma_{-}^{(\ell)} \zeta^{j}\left(b_{j}^{i}\right)^{(\ell)}-\gamma_{+}^{(\ell)} \zeta^{i+1} \mu_{i, j}^{(\ell)}\left(b_{j+1}^{i+1}\right)^{(\ell)}+\lambda_{i, j}^{(\ell)}\left(b_{j+1}^{i}\right)^{(\ell)},
\end{aligned}
$$

where $\mu_{i, j}^{(\ell)}, \lambda_{i, j}^{(\ell)} \in \mathbb{k}$ are scalars satisfying the conditions of Theorems 6.1 and 6.3.

Thus, the desired extended action of the $n$-th Taft algebra on $\mathbb{k} Q$ is complete by the five steps above.

7C. Examples. In this section, we illustrate the algorithm of the previous section to get actions of $T(n)$ on $\mathbb{k} Q$ for various quivers $Q$ having $\mathbb{Z}_{n}$ symmetry.

For ease of exposition, we take all parameters $\mu_{i, j}$ equal to 1 in this section.

Example 7.8. Consider the following quiver $Q$ :

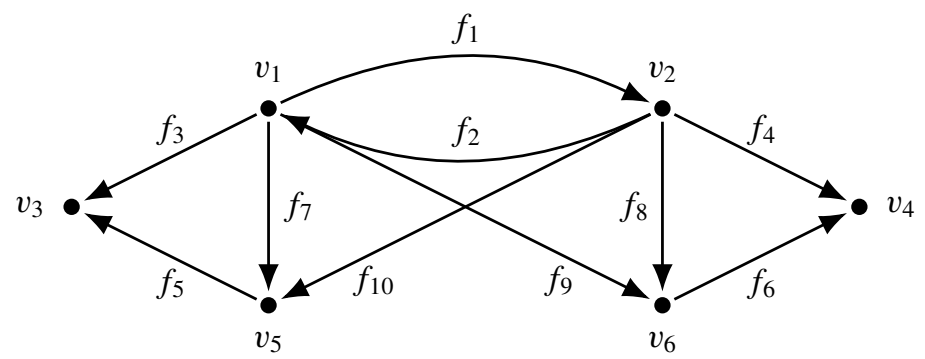

which has $\mathbb{Z}_{2}$-symmetry by reflection over the central vertical axis and exchanging arrows $f_{1}$ and $f_{2}$. So, we decompose $Q$ into the four $\mathbb{Z}_{2}$-components as follows. 
Here, the dotted red arrows indicate the action of $\mathbb{Z}_{2}$ on $Q_{0}$.

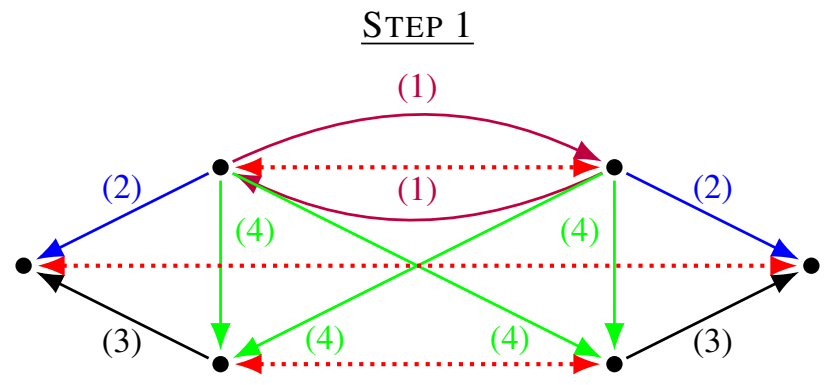

Now choose scalars $\gamma^{(1)}, \gamma_{+}^{(2)}, \gamma_{-}^{(2)}, \gamma_{+}^{(3)}, \gamma_{-}^{(3)}, \gamma_{+}^{(4)}, \gamma_{-}^{(4)} \in \mathbb{k}$ to execute Steps 2 and 3 .

\section{$\underline{\text { STEPS } 2 \text { AND } 3}$}

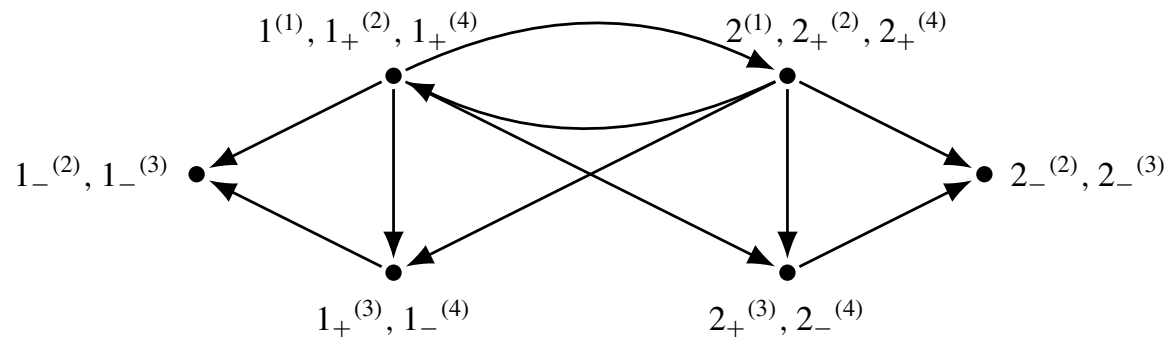

We have

$$
x \cdot e_{1}^{(1)}=-\gamma^{(1)}\left(e_{1}^{(1)}+e_{2}^{(1)}\right), \quad x \cdot e_{2}^{(1)}=\gamma^{(1)}\left(e_{2}^{(1)}+e_{1}^{(1)}\right),
$$

while, for $\ell=2,3,4$,

$$
\begin{array}{ll}
x \cdot e_{1_{+}}^{(\ell)}=-\gamma_{+}^{(\ell)}\left(e_{1_{+}}^{(\ell)}+e_{2_{+}}^{(\ell)}\right), & x \cdot e_{2_{+}}^{(\ell)}=\gamma_{+}^{(\ell)}\left(e_{2_{+}}^{(\ell)}+e_{1_{+}}^{(\ell)}\right), \\
x \cdot e_{1_{-}}^{(\ell)}=-\gamma_{-}^{(\ell)}\left(e_{1_{-}}^{(\ell)}+e_{2_{-}}^{(\ell)}\right), & x \cdot e_{2_{-}}^{(\ell)}=\gamma_{-}^{(\ell)}\left(e_{2_{-}}^{(\ell)}+e_{1_{-}}^{(\ell)}\right) .
\end{array}
$$

By using the identification of vertices

$$
\begin{array}{ll}
v_{1}:=1^{(1)}=1_{+}{ }^{(2)}=1_{+}{ }^{(4)}, & v_{2}:=2^{(1)}=2_{+}{ }^{(2)}=2_{+}{ }^{(4)}, \\
v_{3}:=1_{-}{ }^{(2)}=1_{-}{ }^{(3)}, & v_{4}:=2_{-}{ }^{(2)}=2_{-}{ }^{(3)}, \\
v_{5}:=1_{+}{ }^{(3)}=1_{-}{ }^{(4)}, & v_{6}:=2_{+}{ }^{(3)}=2_{-}{ }^{(4)},
\end{array}
$$

we have

$$
\gamma:=\gamma^{(1)}=\gamma_{+}^{(2)}=\gamma_{+}^{(4)}, \quad \gamma^{\prime}:=\gamma_{-}^{(2)}=\gamma_{-}^{(3)}, \quad \gamma^{\prime \prime}:=\gamma_{+}^{(3)}=\gamma_{-}^{(4)} .
$$


Now, we need to label the arrows of $Q$ appropriately, and invoke Theorems 6.1 and 6.3 to get the desired action of $T(2)$ on $\mathbb{k} Q_{1}$.

\section{$\underline{\text { STEPS } 4 \text { AND } 5}$}

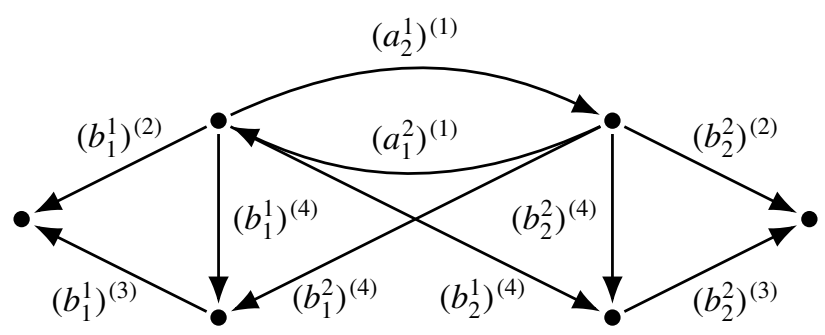

$$
\begin{aligned}
& x \cdot\left(a_{j}^{i}\right)^{(1)}=\gamma^{(1)}\left(\zeta^{j}\left(a_{j}^{i}\right)^{(1)}-\zeta^{i+1}\left(a_{j+1}^{i+1}\right)^{(1)}\right)+\lambda_{i, j}^{(1)}\left(a_{j+1}^{i}\right)^{(1)}, \\
& x \cdot\left(b_{j}^{i}\right)^{(\ell)}=\gamma_{-}^{(\ell)} \zeta^{j}\left(b_{j}^{i}\right)^{(\ell)}-\gamma_{+}^{(\ell)} \zeta^{i+1}\left(b_{j+1}^{i+1}\right)^{(\ell)}+\lambda_{i, j}^{(\ell)}\left(b_{j+1}^{i}\right)^{(\ell)}, \quad \ell=2,3,4 .
\end{aligned}
$$

Using the conditions on the scalars from Theorems 6.1 and 6.3, we find that the $x$-action on the arrows is controlled by additional parameters $\lambda:=\lambda_{1,2}^{(1)}$ as $\sigma(a)$ exists for component (1), and $\lambda^{\prime}:=\lambda_{1,1}^{(4)}, \lambda^{\prime \prime}:=\lambda_{1,2}^{(4)}$ as $\sigma(a)$ exists for component (4). Putting this all together, the action of $\mathbb{Z}_{2}$ on $Q$ (where by abuse of notation, $v_{i}$ denotes the trivial path at $v_{i}$ ) given by

$$
\begin{array}{llll}
g \cdot v_{1}=v_{2}, & g \cdot v_{2}=v_{1}, & g \cdot f_{1}=f_{2}, & g \cdot f_{2}=f_{1}, \\
g \cdot v_{3}=v_{4}, & g \cdot v_{4}=v_{3}, & g \cdot f_{3}=f_{4}, & g \cdot f_{4}=f_{3}, \\
g \cdot v_{5}=v_{6}, & g \cdot v_{6}=v_{5}, & g \cdot f_{5}=f_{6}, & g \cdot f_{6}=f_{5}, \\
& g \cdot f_{7}=f_{8}, & g \cdot f_{8}=f_{7}, \\
& g \cdot f_{9}=f_{10}, & g \cdot f_{10}=f_{9},
\end{array}
$$

extends to an action of the Sweedler algebra on $\mathbb{k} Q$, as follows:

$$
\begin{array}{ll}
x \cdot v_{1}=-\gamma\left(v_{1}+v_{2}\right), & x \cdot v_{2}=\gamma\left(v_{2}+v_{1}\right), \\
x \cdot v_{3}=-\gamma^{\prime}\left(v_{3}+v_{4}\right), & x \cdot v_{4}=\gamma^{\prime}\left(v_{4}+v_{3}\right), \\
x \cdot v_{5}=-\gamma^{\prime \prime}\left(v_{5}+v_{6}\right), & x \cdot v_{6}=\gamma^{\prime \prime}\left(v_{6}+v_{5}\right),
\end{array}
$$

$$
\begin{array}{ll}
x \cdot f_{1}=\gamma f_{1}-\gamma f_{2}+\lambda e_{1}, & x \cdot f_{2}=\gamma f_{1}-\gamma f_{2}-\lambda e_{2}, \\
x \cdot f_{3}=-\gamma^{\prime} f_{3}-\gamma f_{4}, & x \cdot f_{4}=\gamma^{\prime} f_{4}+\gamma f_{3}, \\
x \cdot f_{5}=-\gamma^{\prime} f_{5}-\gamma^{\prime \prime} f_{6}, & x \cdot f_{6}=\gamma^{\prime} f_{6}+\gamma^{\prime \prime} f_{5}, \\
x \cdot f_{7}=-\gamma^{\prime \prime} f_{7}-\gamma f_{8}+\lambda^{\prime} f_{9}, & x \cdot f_{8}=\gamma^{\prime \prime} f_{8}+\gamma f_{7}-\lambda^{\prime} f_{10}, \\
x \cdot f_{9}=\gamma^{\prime \prime} f_{9}-\gamma f_{10}+\lambda^{\prime \prime} f_{7}, & x \cdot f_{10}=-\gamma^{\prime \prime} f_{10}+\gamma f_{9}-\lambda^{\prime \prime} f_{8},
\end{array}
$$

for any scalars $\gamma, \gamma^{\prime}, \gamma^{\prime \prime}, \lambda, \lambda^{\prime}, \lambda^{\prime \prime} \in \mathbb{k}$ which satisfy $(\gamma)^{2}=\left(\gamma^{\prime \prime}\right)^{2}+\lambda^{\prime} \lambda^{\prime \prime}$.

Note that the Sweedler algebra action restricted to the path algebras of respective components (1), (2), (3), (4) is the same as the Sweedler algebra action on the path 
algebra of respective quivers $(\mathrm{I}),(\mathrm{V}),(\mathrm{V}),(\mathrm{VI})$ from Theorem 5.1, as expected; namely, $Q=Q(\mathrm{l}) \circledast Q(\mathrm{~V}) \circledast Q(\mathrm{~V}) \circledast Q(\mathrm{Vl})$.

Example 7.9. Consider the following quiver $Q$ :

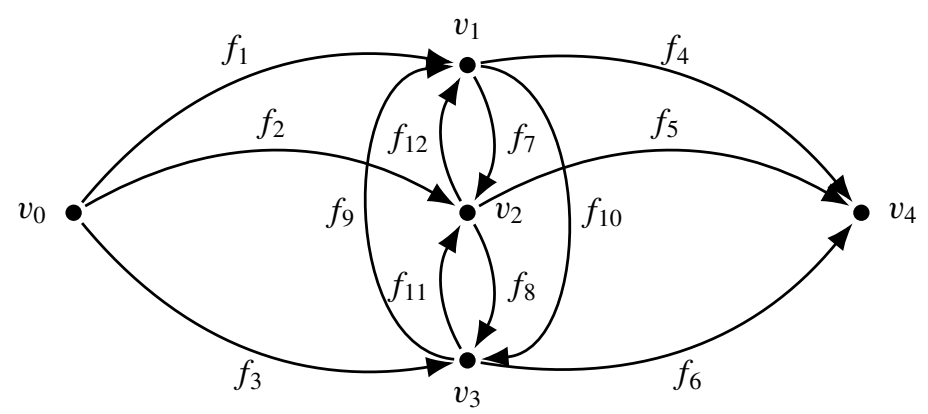

which has $\mathbb{Z}_{3}$-symmetry. We decompose $Q$ into three $\mathbb{Z}_{3}$-components as follows. Here, the dotted red arrows indicate the action of $\mathbb{Z}_{3}$ on $Q_{0}$.

\section{STEP 1}

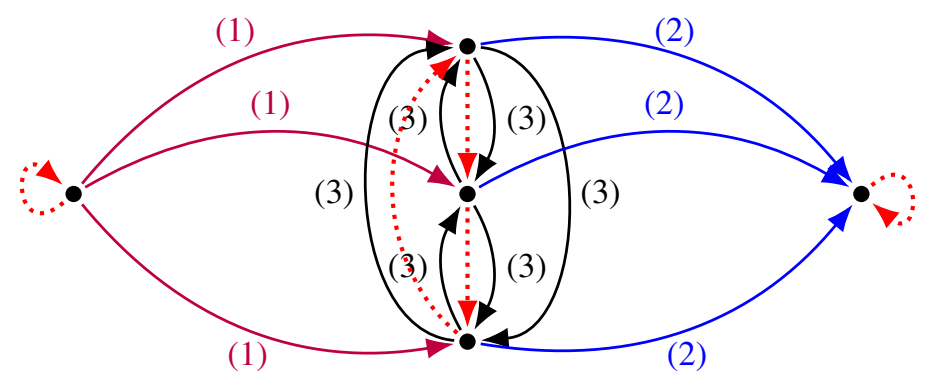

Now choose scalars $\gamma_{+}^{(1)}, \gamma_{-}^{(1)}, \gamma_{+}^{(2)}, \gamma_{-}^{(2)}, \gamma^{(3)} \in \mathbb{k}$ to execute Steps 2 and 3. Further, let $\omega$ be a primitive third root of unity.

\section{STEPS 2 AND 3}

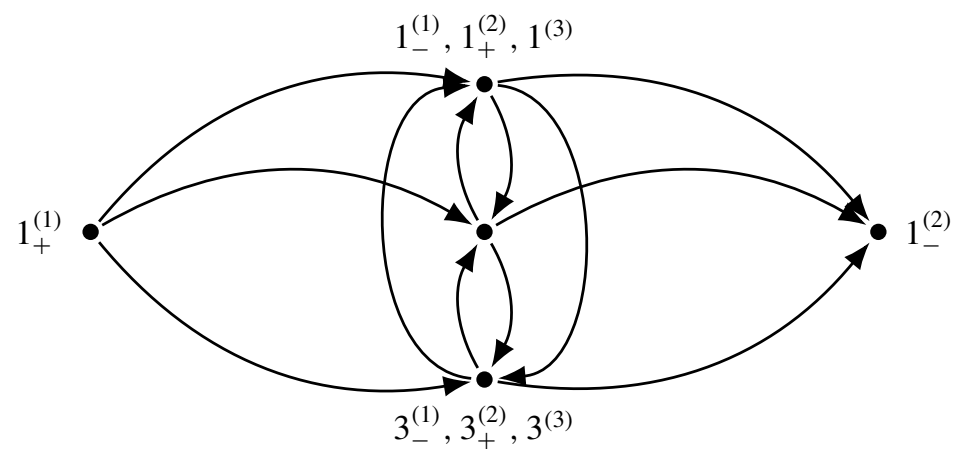

(the middle vertex is labeled by $2_{-}^{(1)}, 2_{+}^{(2)}, 2^{(3)}$ ) 
We have $x \cdot e_{1_{+}}^{(1)}=x \cdot e_{1_{-}}^{(2)}=0$ since these vertices are fixed by $g$, and furthermore

$$
\begin{gathered}
x \cdot e_{1_{-}}^{(1)}=\gamma_{-}^{(1)} \omega\left(e_{1_{-}}^{(1)}-\omega e_{2_{-}}^{(1)}\right), \quad x \cdot e_{1_{+}}^{(2)}=\gamma_{+}^{(2)} \omega\left(e_{1_{+}}^{(2)}-\omega e_{2_{+}}^{(2)}\right), \\
x \cdot e_{2_{-}}^{(1)}=\gamma_{-}^{(1)} \omega^{2}\left(e_{2_{-}}^{(1)}-\omega e_{3_{-}}^{(1)}\right), \quad x \cdot e_{2_{+}}^{(2)}=\gamma_{+}^{(2)} \omega^{2}\left(e_{2_{+}}^{(2)}-\omega e_{3_{+}}^{(2)}\right), \\
x \cdot e_{3_{-}}^{(1)}=\gamma_{-}^{(1)}\left(e_{3_{-}}^{(1)}-\omega e_{1_{-}}^{(1)}\right), \quad x \cdot e_{3_{+}}^{(2)}=\gamma_{+}^{(2)}\left(e_{3_{+}}^{(2)}-\omega e_{1_{+}}^{(2)}\right) \\
x \cdot e_{1}^{(3)}=\gamma^{(3)} \omega\left(e_{1}^{(3)}-\omega e_{2}^{(3)}\right) \\
x \cdot e_{2}^{(3)}=\gamma^{(3)} \omega^{2}\left(e_{2}^{(3)}-\omega e_{3}^{(3)}\right) \\
x \cdot e_{3}^{(3)}=\gamma^{(3)}\left(e_{3}^{(3)}-\omega e_{1}^{(3)}\right)
\end{gathered}
$$

By using the identification of vertices,

$$
v_{1}=1_{-}^{(1)}=1_{+}^{(2)}=1^{(3)}, \quad v_{2}=2_{-}^{(1)}=2_{+}^{(2)}=2^{(3)}, \quad v_{3}=3_{-}^{(1)}=3_{+}^{(2)}=3^{(3)},
$$

we have

$$
\gamma:=\gamma_{-}^{(1)}=\gamma_{+}^{(2)}=\gamma^{(3)}, \quad \gamma_{+}^{(1)}=0, \quad \gamma_{-}^{(2)}=0 .
$$

Now, we need to label the arrows of $Q$ appropriately, and invoke Theorems 6.1 and 6.3 to get the desired action of $T(3)$ on $\mathbb{k} Q_{1}$.

\section{$\underline{\text { STEPS } 4 \text { AND } 5}$}

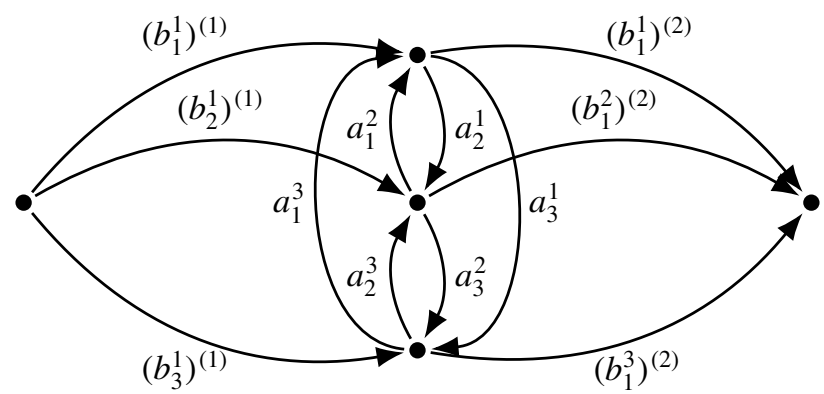

$$
\begin{aligned}
& x \cdot\left(b_{j}^{1}\right)^{(1)}=\gamma \omega^{j}\left(b_{j}^{1}\right)^{(1)}+\lambda \omega^{j-1}\left(b_{j+1}^{1}\right)^{(1)}, \\
& x \cdot\left(b_{1}^{i}\right)^{(2)}=-\gamma \omega^{i+1}\left(b_{1}^{i+1}\right)^{(2)}+\lambda^{\prime} \omega^{i-1}\left(b_{1}^{i}\right)^{(2)}, \\
& x \cdot\left(a_{j}^{i}\right)^{(3)}=\gamma\left(\omega^{j}\left(a_{j}^{i}\right)^{(3)}-\omega^{i+1}\left(a_{j+1}^{i+1}\right)^{(3)}\right)+\lambda_{i, j}^{(3)}\left(a_{j+1}^{i}\right)^{(3)}, \\
& \text { where } \lambda:=\lambda_{1,1}^{(1)} \text { and } \lambda^{\prime}:=\lambda_{1,1}^{(2)} .
\end{aligned}
$$

Moreover, let $\lambda^{\prime \prime}:=\lambda_{1,2}^{(3)}$ and $\lambda^{\prime \prime \prime}:=\lambda_{1,3}^{(3)}$ for component (3). Putting this all together, the action of $\mathbb{Z}_{3}$ on $Q$ given by Step 1 extends to an action of $T(3)$ on $\mathbb{k} Q$, as 
follows:

$$
\begin{array}{lll}
x \cdot v_{1}=\gamma \omega\left(v_{1}-\omega v_{2}\right), & x \cdot v_{2}=\gamma \omega^{2}\left(v_{2}-\omega v_{3}\right), & x \cdot v_{3}=\gamma\left(v_{3}-\omega v_{1}\right), \\
x \cdot v_{0}=0, & x \cdot v_{4}=0, & \\
x \cdot f_{1}=\gamma \omega f_{1}+\lambda f_{2}, & x \cdot f_{4}=\lambda^{\prime} f_{4}-\gamma \omega^{2} f_{5}, \\
x \cdot f_{2}=\gamma \omega^{2} f_{2}+\lambda \omega f_{3}, & x \cdot f_{5}=\lambda^{\prime} \omega f_{5}-\gamma f_{6}, \\
x \cdot f_{3}=\gamma f_{3}+\lambda \omega^{2} f_{1}, & x \cdot f_{6}=\lambda^{\prime} \omega^{2} f_{6}-\gamma \omega f_{4}, \\
x \cdot f_{7}=\gamma\left(\omega^{2} f_{7}-\omega^{2} f_{8}\right)+\lambda^{\prime \prime} f_{10}, & x \cdot f_{10}=\gamma\left(f_{10}-\omega^{2} f_{12}\right)+\lambda^{\prime \prime \prime} f_{7}, \\
x \cdot f_{8}=\gamma\left(f_{8}-f_{9}\right)+\lambda^{\prime \prime} \omega f_{12}, & x \cdot f_{11}=\gamma\left(\omega^{2} f_{11}-\omega f_{10}\right)+\lambda^{\prime \prime \prime} \omega f_{9}, \\
x \cdot f_{9}=\gamma\left(\omega f_{9}-\omega f_{7}\right)+\lambda^{\prime \prime} \omega^{2} f_{11}, & x \cdot f_{12}=\gamma\left(\omega f_{12}-f_{11}\right)+\lambda^{\prime \prime \prime} \omega^{2} f_{8},
\end{array}
$$

for any scalars $\gamma, \lambda, \lambda^{\prime}, \lambda^{\prime \prime}, \lambda^{\prime \prime \prime} \in \mathbb{k}$.

\section{Extended actions of other pointed Hopf algebras on path algebras}

In this section, we extend the results in the previous sections to actions of the Frobenius-Lusztig kernel (Section 8A) and to actions of the Drinfeld double of a Taft algebra (Section 8B). We remind the reader of the standing assumptions made in Hypothesis 1.2.

Notation 8.1 $(q, T(n, \xi))$. Let $q \in \mathbb{k}$ be a primitive $2 n$-th root of unity, and let $\xi \in \mathbb{k}$ be a primitive $n$-th root of unity. Moreover, let $T(n, \xi)$ be the Taft algebra generated by a grouplike element $g$ and a $(1, g)$-skew primitive element $x$, subject to the relations $g^{n}=1, x^{n}=0, x g=\xi g x$. Note that $T(n, \zeta)=T(n)$ as in Definition 2.1, for $\zeta$ the fixed primitive $n$-th root of unity of this work.

\section{A. Actions of $\boldsymbol{u}_{q}\left(\mathfrak{s l}_{2}\right)$.}

Definition 8.2 $\left(u_{q}\left(\mathfrak{s l}_{2}\right)\right.$, Borel subalgebras $u_{q}^{\geq 0}\left(\mathfrak{s l}_{2}\right)$ and $\left.u_{q}^{\leq 0}\left(\mathfrak{s l}_{2}\right)\right)$. The FrobeniusLusztig kernel $u_{q}\left(\mathfrak{s l}_{2}\right)$ is the Hopf algebra generated by a grouplike element $K$, a $(1, K)$-skew-primitive element $E$, and a $\left(K^{-1}, 1\right)$-skew-primitive element $F$, subject to the relations

$$
\begin{gathered}
K E=q^{2} E K, \quad K F=q^{-2} F K, \quad K^{n}=1, \quad E^{n}=F^{n}=0, \\
E F-F E=\frac{K-K^{-1}}{q-q^{-1}} .
\end{gathered}
$$

Note that $u_{q}\left(\mathfrak{s l}_{2}\right)$ is pointed, of dimension $n^{3}$. Let $u_{q}^{\geq 0}\left(\mathfrak{s l}_{2}\right)$ be the Hopf subalgebra of $u_{q}\left(\mathfrak{s l}_{2}\right)$ generated by $K, E$, and let $u_{q}^{\leq 0}\left(\mathfrak{s l}_{2}\right)$ be the Hopf subalgebra of $u_{q}\left(\mathfrak{s l}_{2}\right)$ generated by $K, F$; we refer to these as Borel subalgebras.

Lemma 8.4. There are isomorphisms of Hopf algebras

$$
u_{q}^{\geq 0}\left(\mathfrak{s l}_{2}\right) \simeq T\left(n, q^{-2}\right) \quad \text { and } \quad u_{q}^{\leq 0}\left(\mathfrak{s l}_{2}\right) \simeq T\left(n, q^{2}\right) .
$$


Proof. This is easy to check: identify $K$ with $g$ for both $u_{q}^{\geq 0}\left(\mathfrak{s l}_{2}\right)$ and $u_{q}^{\leq 0}\left(\mathfrak{s l}_{2}\right)$, and $E$ with $x$ for $u_{q}^{\geq 0}\left(\mathfrak{s l}_{2}\right)$, and $F$ with $g^{-1} x$ for $u_{q}^{\leq 0}\left(\mathfrak{s l}_{2}\right)$.

Since $u_{q}\left(\mathfrak{s l}_{2}\right)$ is generated by the Hopf subalgebras $u_{q}^{\geq 0}\left(\mathfrak{s l}_{2}\right)$ and $u_{q}^{\leq 0}\left(\mathfrak{s l}_{2}\right)$, an action of $u_{q}\left(\mathfrak{s l}_{2}\right)$ on a path algebra $\mathbb{k} Q$ is determined by its restriction to these Hopf subalgebras. Since these actions are given by the results on Taft actions on $\mathbb{k} Q$ in the previous sections, we can classify actions of $u_{q}\left(\mathfrak{s l}_{2}\right)$ by determining some additional compatibility conditions on the scalar parameters from these Taft actions. We begin with the result below.

Proposition 8.6. Let $u_{q}\left(\mathfrak{s l}_{2}\right)$ act on the path algebra of a set of vertices $\{1, \ldots, m\}$, labeled so that $K \cdot e_{i}=e_{i+1}$ with subscripts taken modulo $m$.

(i) If $m<n$, then $m=1$ or $m=2$ and the action factors through the quotient $u_{q}\left(\mathfrak{s l}_{2}\right) /\langle E, F\rangle$.

(ii) If $m=n$, then

$$
E \cdot e_{i}=\gamma^{E} q^{-2 i}\left(e_{i}-q^{-2} e_{i+1}\right), \quad F \cdot e_{i}=\gamma^{F} q^{2 i}\left(e_{i-1}-q^{2} e_{i}\right),
$$

for some $\gamma^{E}, \gamma^{F} \in \mathbb{k}$. If furthermore $n \geq 3$, then the action is subject to the restriction

$$
-\gamma^{E} \gamma^{F} q^{-1}\left(q^{2}-1\right)^{2}=1 .
$$

Proof. Part (i) is a result of Proposition 3.5, translated through the isomorphisms of Lemma 8.4. The condition $m=1$ or $m=2$ comes from the relation (8.3), which implies that $K-K^{-1}$ acts by zero in this case. For (ii), Proposition 3.5 also gives the formulas (8.7), and the first row of relations in the definition of $u_{q}\left(\mathfrak{s l}_{2}\right)$ then hold. On the other hand, substituting (8.7) into the relation (8.3) of $u_{q}\left(\mathfrak{s l}_{2}\right)$ yields $(E F-F E) \cdot e_{i}=\gamma^{E} \gamma^{F}\left(q^{2}-1\right)\left(e_{i-1}-e_{i+1}\right) \quad$ while $\left(K-K^{-1}\right) \cdot e_{i}=e_{i+1}-e_{i-1}$. If $n=m=2$, then $e_{i-1}=e_{i+1}$ and the relation (8.3) imposes no restriction on the action (8.7). If $n=m \geq 3$, then $e_{i+1} \neq e_{i-1}$ and the relation (8.3) imposes the restriction (8.8) on the action (8.7).

We get the following immediate consequence.

Corollary 8.9. If $n \geq 3$, then either one of $\gamma^{E}$ or $\gamma^{F}$ determines the other. So, an action of $u_{q}\left(\mathfrak{s l}_{2}\right)$ on a $\mathbb{Z}_{n}$-orbit of vertices is completely determined by the action of a Borel subalgebra, $u_{q}^{\geq 0}\left(\mathfrak{s l}_{2}\right)$ or $u_{q}^{\leq 0}\left(\mathfrak{s l}_{2}\right)$.

We now consider $u_{q}\left(\mathfrak{s l}_{2}\right)$-actions on Type A and Type B $\mathbb{Z}_{n}$-minimal quivers. Note that $u_{q}\left(\mathfrak{s l}_{2}\right)$ can only act on subquivers of $K_{m}$ and $K_{m, m^{\prime}}$ for $m, m^{\prime} \in\{1,2, n\}$, by Proposition 8.6. We only give theorems describing the case $m=m^{\prime}=n \geq 3$ here, but the other cases can be worked out similarly. 
Theorem 8.10. Retain the notation of Section 4 with $K$ in place of $g$, so the action of $K$ is fixed. Namely, $K \cdot a_{j}^{i}=\mu_{i, j} a_{j+1}^{i+1}$ for Type $A$ and $K \cdot b_{j}^{i}=\mu_{i, j} b_{j+1}^{i+1}$ for Type B. Assume $n \geq 3$. Then any $u_{q}\left(\mathfrak{s l}_{2}\right)$-action on the path algebra of a $\mathbb{Z}_{n}$-minimal subquiver $Q$ of $K_{n}$ is given by

$$
\begin{aligned}
& E \cdot a_{j}^{i}=\gamma^{E}\left(q^{-2 j} a_{j}^{i}-q^{-2 i-2} a_{j+1}^{i+1}\right)+\lambda_{i, j}^{E} a_{j+1}^{i}, \\
& F \cdot a_{j}^{i}=\gamma^{F}\left(q^{2 j} a_{j-1}^{i-1}-q^{2 i+2} a_{j}^{i}\right)+\lambda_{i, j}^{F} a_{j}^{i-1},
\end{aligned}
$$

subject to the restriction (8.8) along with

- $\lambda_{i, j}^{E}=0$, if $i=j$ or the arrow $a_{j+1}^{i}$ does not exist in $Q$;

- $\lambda_{i, j}^{F}=0$, if $i=j$ or the arrow $a_{j}^{i-1}$ does not exist in $Q$;

- $\lambda_{i+1, j+1}^{E}=q^{-2} \lambda_{i, j}^{E}, \quad \lambda_{i+1, j+1}^{F}=q^{2} \lambda_{i, j}^{F}, \quad$ and $\quad \lambda_{i, j}^{F} \lambda_{i-1, j}^{E}=\lambda_{i, j}^{E} \lambda_{i, j+1}^{F}$.

Any $u_{q}\left(\mathfrak{s l}_{2}\right)$-action on the path algebra of a $\mathbb{Z}_{n}$-minimal subquiver $Q$ of $K_{n, n}$ is given by

$$
\begin{aligned}
& E \cdot b_{j}^{i}=\left(\gamma_{-}^{E}\right) q^{-2 j} b_{j}^{i}-\left(\gamma_{+}^{E}\right) q^{-2 i-2} b_{j+1}^{i+1}+\lambda_{i, j}^{E} b_{j+1}^{i}, \\
& F \cdot a_{j}^{i}=\left(\gamma_{-}^{F}\right) q^{2 j} b_{j-1}^{i-1}-\left(\gamma_{+}^{F}\right) q^{2 i+2} b_{j}^{i}+\lambda_{i, j}^{F} b_{j}^{i-1},
\end{aligned}
$$

subject to restrictions of the form (8.8) on $\gamma_{ \pm}^{E}$ and $\gamma_{ \pm}^{F}$, along with

- $\lambda_{i, j}^{E}=0$, if $i=j$ or the arrow $b_{j+1}^{i}$ does not exist in $Q$;

- $\lambda_{i, j}^{F}=0$, if $i=j$ or the arrow $b_{j}^{i-1}$ does not exist in $Q$;

- $\lambda_{i+1, j+1}^{E}=q^{-2} \lambda_{i, j}^{E}, \quad \lambda_{i+1, j+1}^{F}=q^{2} \lambda_{i, j}^{F}, \quad$ and $\quad \lambda_{i, j}^{F} \lambda_{i-1, j}^{E}=\lambda_{i, j}^{E} \lambda_{i, j+1}^{F}$;

- $\left(\gamma_{+}^{E}\right)^{n}=\left(\gamma_{-}^{E}\right)^{n}+\prod_{\ell=0}^{n-1} \lambda_{i, j+\ell}^{E} \quad$ and $\quad\left(\gamma_{+}^{F}\right)^{n}=\left(\gamma_{-}^{F}\right)^{n}+\prod_{\ell=0}^{n-1} \lambda_{i, j+\ell}^{F}$.

Proof. First consider the Type A case. Proposition 8.6 gives the $u_{q}\left(\mathfrak{s l}_{2}\right)$-action on the vertices, imposing the restriction (8.8). Theorem 6.1, translated through the isomorphisms of Lemma 8.4, initially gives expressions for $E \cdot a_{j}^{i}$ and $F \cdot a_{j}^{i}$ which involve parameters $\mu_{i, j}$. However, we examine the coefficients of various arrows in the relation (8.3) applied to $a_{j}^{i}$, and find that the coefficient of $a_{j-1}^{i-1}$ gives

$$
\gamma^{E} \gamma^{F}\left(q^{2}-1\right)+\mu_{i, j}\left(q-q^{-1}\right)^{-1}=0 .
$$

By applying (8.8), this simplifies to $\left(\mu_{i, j}-1\right)\left(q-q^{-1}\right)^{-1}=0$. So, $\mu_{i, j}=1$ for all $i, j$, which gives our formulas (8.11). Similarly, the coefficient of $a_{j+1}^{i-1}$ in the relation (8.3) applied to $a_{j}^{i}$ implies that

$$
\lambda_{i, j}^{F} \lambda_{i-1, j}^{E}-\lambda_{i, j}^{E} \lambda_{i, j+1}^{F}=0 .
$$

Theorem 6.1 gives the remaining restrictions on the scalars, and the coefficients of other arrows yield restrictions that are already implied by those above.

For Type B, the action is derived exactly as in the Type A case, by replacing $a_{\star}^{\star}$ with $b_{\star}^{\star}$, and $\gamma$ with $\gamma_{ \pm}$, appropriately. 
8B. Actions of the double $D(T(n))$. We take the presentation of the Drinfeld double of the $n$-th Taft algebra in [Chen 1999, Definition-Theorem 3.1]. Namely, by [Chen 1999, Theorem 3.3], the double is isomorphic to the Hopf algebra $H_{n}(p, q)$ generated by $a, b, c, d$. We take $p=1, q=\zeta^{-1}, a=x, b=g, c=G$, and $d=X$ to get that:

Definition $8.15(D(T(n)))$. The Drinfeld double $D(T(n))$ of the $n$-th Taft algebra is generated by $g, x, G, X$, subject to relations

$$
\begin{array}{rlll}
x g & =\zeta g x, & g X=\zeta X g, & g^{n}=G^{n}=1, \\
G X=\zeta X G, & x G=\zeta G x, & x^{n}=X^{n}=0, & g G=G g,
\end{array}
$$

and

$$
x X-\zeta X x=\zeta(g G-1)
$$

Here, $g$ and $G$ are grouplike elements, $x$ is $(1, g)$-a skew primitive element, and $X$ is a $(1, G)$-skew primitive element. Here, $g, x$ generate the Hopf subalgebra $T(n)$, and $G=g^{*}, X=x^{*}$ generate $\left(T(n)^{\text {op }}\right)^{*}=\left(T(n)^{*}\right)^{\text {cop }}$.

The following lemma is easy to check.

Lemma 8.17. The Hopf subalgebra of $D(T(n))$ generated by $g, x$ is isomorphic to $T(n)=T(n, \zeta)$, as Hopf algebras. Moreover, the Hopf subalgebra of $D(T(n))$ generated by $G, X$ is isomorphic to $T\left(n, \zeta^{-1}\right)$, as Hopf algebras.

To give the complete classification of $D(T(n))$-actions on path algebras of quivers, one would need to define $\mathbb{Z}_{n} \times \mathbb{Z}_{n}$-minimal quivers and consider the $D(T(n))$-action on these, since $\mathbb{Z}_{n} \times \mathbb{Z}_{n}$ is isomorphic to the group of grouplike elements of $D(T(n))$. This is the subject of future work. Our aim for now is to exhibit an action of $D(T(n))$ on $\mathbb{k} Q$, where $Q$ admits an action of $\mathbb{Z}_{n}$. We begin by providing an action of $D(T(n))$ on a $\mathbb{Z}_{n}$-orbit of vertices.

Proposition 8.18. An action of $D(T(n))$ on $\mathbb{k} Q_{0}$ where $Q_{0}=\{1, \ldots, n\}$ is given by

$$
\begin{aligned}
g \cdot e_{i} & =e_{i+1}, \quad x \cdot e_{i}=\gamma^{x} \zeta^{i}\left(e_{i}-\zeta e_{i+1}\right), \\
G \cdot e_{i} & =e_{i+1}, \quad X \cdot e_{i}=\gamma^{X} \zeta^{-i}\left(e_{i}-\zeta^{-1} e_{i+1}\right)
\end{aligned}
$$

for some $\gamma^{x}, \gamma^{X} \in \mathbb{k}$. If $n \geq 3$, then these scalars are subject to the restriction

$$
\gamma^{x} \gamma^{X}\left(1-\zeta^{-1}\right)=1
$$

Proof. The formulas (8.19) satisfy the first two rows of relations of $D(T(n))$. On the other hand, the restriction (8.20) comes from substituting (8.19) into the relation (8.16) of $D(T(n))$ when $n \geq 3$. If $n=2$, the restriction from (8.16) is vacuous. 
We proceed by extending the $D(T(n))$-action on vertices in Proposition 8.18 to yield an action of $D(T(n))$ on Type A and Type B $\mathbb{Z}_{n}$-minimal quivers. As in the $u_{q}\left(\mathfrak{s l}_{2}\right)$ case, we only give theorems describing the cases when each orbit of vertices has $n$ elements here.

Theorem 8.21. Retain the notation of Section 4. An action of $D(T(n))$ on the path algebra of a Type $A \mathbb{Z}_{n}$-minimal subquiver $Q$ of $K_{n}$ is given by

$$
\begin{aligned}
& g \cdot a_{j}^{i}=\mu_{i, j}^{g} a_{j+1}^{i+1}, \quad x \cdot a_{j}^{i}=\gamma^{x}\left(\zeta^{j} a_{j}^{i}-\zeta^{i+1} \mu_{i, j}^{g} a_{j+1}^{i+1}\right)+\lambda_{i, j}^{x} a_{j+1}^{i}, \\
& G \cdot a_{j}^{i}=\mu_{i, j}^{G} a_{j+1}^{i+1}, \quad X \cdot a_{j}^{i}=\gamma^{X}\left(\zeta^{-j} a_{j}^{i}-\zeta^{-i-1} \mu_{i, j}^{G} a_{j+1}^{i+1}\right)+\lambda_{i, j}^{X} a_{j+1}^{i},
\end{aligned}
$$

subject to the restrictions

$$
\begin{gathered}
\mu_{i, i}^{g}=\mu_{i, i}^{G}=1 \text { for all } i, \\
\prod_{\ell=0}^{n-1} \mu_{i+\ell, j+\ell}^{g}=\prod_{\ell=0}^{n-1} \mu_{i+\ell, j+\ell}^{G}=1, \quad \mu_{i, j}^{G} \mu_{i+1, j+1}^{g}=\mu_{i, j}^{g} \mu_{i+1, j+1}^{G}, \\
\zeta \mu_{i, j+1}^{g} \lambda_{i, j}^{x}=\mu_{i, j}^{g} \lambda_{i+1, j+1}^{x}, \quad \zeta \mu_{i, j+1}^{G} \lambda_{i, j}^{X}=\mu_{i, j}^{G} \lambda_{i+1, j+1}^{X}, \\
\zeta \mu_{i, j}^{g} \lambda_{i+1, j+1}^{X}=\mu_{i, j+1}^{g} \lambda_{i, j}^{X}, \quad \zeta \mu_{i, j+1}^{G} \lambda_{i, j}^{x}=\mu_{i, j}^{G} \lambda_{i+1, j+1}^{x}, \\
\lambda_{i, j}^{X} \lambda_{i, j+1}^{x}-\zeta \lambda_{i, j}^{x} \lambda_{i, j+1}^{X}=0, \\
\lambda_{i, j}^{x}=\lambda_{i, j}^{X}=0 \text { if either } i=j, \text { or the arrow } a_{j+1}^{i} \text { does not exist in } Q .
\end{gathered}
$$

If $n \geq 3$, then we also impose the condition $\gamma^{x} \gamma^{X}\left(1-\zeta^{-1}\right)=1$.

An action of $D(T(n))$ on the path algebra of a Type $B$ minimal subquiver $Q$ of $K_{n, n}$ is given by

$$
\begin{aligned}
& g \cdot b_{j}^{i}=\mu_{i, j}^{g} b_{j+1}^{i+1}, \quad G \cdot b_{j}^{i}=\mu_{i, j}^{G} b_{j+1}^{i+1}, \\
& x \cdot b_{j}^{i}=\left(\gamma_{-}^{x}\right) \zeta^{j} b_{j}^{i}-\left(\gamma_{+}^{x}\right) \zeta^{i+1} \mu_{i, j}^{g} b_{j+1}^{i+1}+\lambda_{i, j}^{x} b_{j+1}^{i}, \\
& X \cdot b_{j}^{i}=\left(\gamma_{-}^{X}\right) \zeta^{-j} b_{j}^{i}-\left(\gamma_{+}^{X}\right) \zeta^{-i-1} \mu_{i, j}^{G} b_{j+1}^{i+1}+\lambda_{i, j}^{X} b_{j+1}^{i},
\end{aligned}
$$

subject to the restrictions $(*)$. If $n \geq 3$, then we also impose the condition $\left(\gamma_{ \pm}^{x}\right)\left(\gamma_{ \pm}^{X}\right)\left(1-\zeta^{-1}\right)=1$.

Proof. First, Proposition 8.18 gives an $D(T(n))$-action on a $\mathbb{Z}_{n}$-orbit of vertices; this imposes the restriction (8.20) when $n \geq 3$. Now, consider the Type A case. Theorem 6.1 gives the expressions in (8.22), along with some restrictions on parameters, which are listed in the first two rows of restrictions in the statement of the theorem. Now we need to check that the expressions, $g \cdot a_{j}^{i}, G \cdot a_{j}^{i}, x \cdot a_{j}^{i}, X \cdot a_{j}^{i}$, satisfy the relations of $D(T(n))$. The relations $g G=G g, g X=\zeta X g, x G=\zeta G x$, imply, respectively, that

$\mu_{i, j}^{G} \mu_{i+1, j+1}^{g}=\mu_{i, j}^{g} \mu_{i+1, j+1}^{G}, \zeta \mu_{i, j}^{g} \lambda_{i+1, j+1}^{X}=\mu_{i, j+1}^{g} \lambda_{i, j}^{X}, \quad \zeta \mu_{i, j+1}^{G} \lambda_{i, j}^{x}=\mu_{i, j}^{G} \lambda_{i+1, j+1}^{x}$. 
With these restrictions, it is easy to check that the relation $x X-\zeta X x-\zeta(g G-1)=0$ yields

$$
\lambda_{i, j}^{X} \lambda_{i, j+1}^{x}-\zeta \lambda_{i, j}^{x} \lambda_{i, j+1}^{X}=0,
$$

and we are done with the Type A case. For Type B, the action is derived exactly as in the Type A case, by replacing $a_{\star}^{\star}$ with $b_{\star}^{\star}$, and $\gamma$ with $\gamma_{ \pm}$, appropriately.

8C. Gluing. Gluing is achieved in the following manner. Let $Q$ be a quiver that admits an action of $\mathbb{Z}_{n}$. Since the actions of $u_{q}\left(\mathfrak{s l}_{2}\right)$ and $D(T(n))$ on $\mathbb{k} Q$ in Theorems 8.10 and 8.21 are determined by Taft actions, Theorem 7.5 and the algorithm in Section 7B applies. In other words, we can glue the actions of $u_{q}\left(\mathfrak{s l}_{2}\right)$ or of $D(T(n))$ on path algebras of $\mathbb{Z}_{n}$-minimal quivers. Hence, we obtain an action of $u_{q}\left(\mathfrak{s l}_{2}\right)$ (resp. $D(T(n)))$ extending a Taft action on any path algebra $\mathbb{k} Q$, where each path algebra of a $\mathbb{Z}_{n}$-minimal component of $Q$ admits an action of $u_{q}\left(\mathfrak{s l}_{2}\right)($ resp. $D(T(n)))$.

\section{Appendix}

In this appendix, we show that the relation $x^{n}=0$ imposes no restrictions on the $x$-action on the vertex $e_{i}$ and on the arrows $a_{j}^{i}, b_{j}^{i}$ of $\mathbb{k} Q$, which is used in the proofs of Proposition 3.5 and Theorems 6.1 and 6.3. Recall that $\zeta$ is a primitive $n$-th root of unity, with $n \geq 2$. An easy computation shows that

$$
\prod_{\ell=1}^{n} \zeta^{\ell}=\zeta^{\frac{n(n+1)}{2}}= \begin{cases}1, & n \text { odd, } \\ -1, & n \text { even },\end{cases}
$$

a fact we will use several times here.

This appendix uses some basic properties of symmetric polynomials and $q$ binomial coefficients, which we recall here. For integers $a, b$, the complete symmetric polynomial of degree $a$, denoted $h_{a}\left(x_{0}, \ldots, x_{b}\right)$, is defined as the sum of all monomials of total degree $a$ in the variables $x_{0}, \ldots, x_{b}$. We interpret the variable set as being empty when $b<0$. When $a=0$, we have $h_{0}\left(x_{0}, \ldots, x_{b}\right)=1$ for any integer $b$. When $a \neq 0$, we have $h_{a}\left(x_{0}, \ldots, x_{b}\right)=0$ if $a<0$ or $b<0$. Also, a product $\prod_{i=a}^{b}$ is taken to be 1 whenever $b<a$. These polynomials are homogeneous, meaning that $h_{a}\left(c x_{0}, \ldots, c x_{b}\right)=c^{a} h_{a}\left(x_{0}, \ldots, x_{b}\right)$ for any scalar $c$. We have immediately from the definition that

$$
h_{a}\left(x_{0}, \ldots, x_{b-1}\right)+x_{b} h_{a-1}\left(x_{0}, \ldots, x_{b}\right)=h_{a}\left(x_{0}, \ldots, x_{b}\right)
$$

for any integers $a, b$.

Lemma 9.3. For nonnegative integers $a, b$, we have $h_{a}\left(1, \zeta, \zeta^{2}, \ldots, \zeta^{b}\right)=0$ when $n$ divides $a+b$ and $a, b \neq 0$. 
Proof. Recall that for a nonnegative integer $k$, the corresponding $q$-integer is defined as $[k]_{q}=1+q+q^{2}+\cdots+q^{k-1}$, and that its evaluation $[k]_{q=\zeta}$ is 0 if and only if $n$ divides $k$. The $q$-binomial coefficient $\left[\begin{array}{l}i \\ j\end{array}\right]_{q}$ is defined by a factorial formula analogous to that of binomial coefficients, so the evaluation $\left[\begin{array}{l}i \\ j\end{array}\right]_{q=\zeta}$ vanishes when $n$ divides $i$ and $j \neq 0, i$.

The principal specialization from [Stanley 1999, Proposition 7.8.3], with $q=\zeta$, gives us that

$$
h_{a}\left(1, \zeta, \zeta^{2}, \ldots, \zeta^{b}\right)=\left[\begin{array}{c}
a+b \\
a
\end{array}\right]_{q=\zeta}
$$

so by the paragraph above this quantity vanishes when $n$ divides $a+b$ and $a, b \neq 0$.

We prove a general lemma about a linear operator $X$ acting as the element $x$ does.

Lemma 9.4. Let $V$ be a vector space, and consider a collection of vectors $\left\{v_{j}^{i}\right\}$ in $V$, where $1 \leq i \leq m$ and $1 \leq j \leq m^{\prime}$. Interpret $i$ and $j$ modulo $m$ and $m^{\prime}$ respectively, so that they lie inside their ranges. Let $X$ be a linear operator acting on $V$ such that

$$
X v_{j}^{i}=\eta_{j} v_{j}^{i}+\theta_{i, j} v_{j+1}^{i+1}+\tau_{i, j} v_{j+1}^{i}
$$

for some scalars $\eta_{j}, \theta_{i, j}, \tau_{i, j} \in \mathbb{k}$. Furthermore, assume that the scalars satisfy the relation

$$
\tau_{i+1, j+1} \theta_{i, j}=\zeta \theta_{i, j+1} \tau_{i, j}
$$

for all pairs $(i, j)$. Then, for all positive integers $k$, we have

$$
X^{k}\left(v_{j}^{i}\right)=\sum_{0 \leq s \leq t \leq k} \psi_{k, s, t} v_{j+t}^{i+s}
$$

where

$$
\begin{aligned}
\psi_{k, s, t}=\left(\prod_{\ell=0}^{s-1} \theta_{i+\ell, j+\ell+t-s}\right) & \left(\prod_{\ell=0}^{t-s-1} \tau_{i, j+\ell}\right) \\
& \times h_{k-t}\left(\eta_{j}, \eta_{j+1}, \cdots, \eta_{j+t}\right) h_{s}\left(1, \zeta, \ldots, \zeta^{t-s}\right) .
\end{aligned}
$$

Proof. We proceed by induction on $k$. The base case of $k=1$ follows from simple substitution. So assume that the statement is true when $k$ is replaced by $k-1$; that is, we have a formula for $X^{k-1}\left(v_{j}^{i}\right)$ for all pairs $(i, j)$. Now, when applying $X$ to $X^{k-1}\left(v_{j}^{i}\right)$, the coefficient $\psi_{k, s, t}$ is the sum of contributions from three terms:

- $X\left(\psi_{k-1, s, t} v_{j+t}^{i+s}\right)$ contributes $\eta_{j+t} \psi_{k-1, s, t}$;

- $X\left(\psi_{k-1, s-1, t-1} v_{j+t-1}^{i+s-1}\right)$ contributes $\theta_{i+s-1, j+t-1} \psi_{k-1, s-1, t-1}$;

- $X\left(\psi_{k-1, s, t-1} v_{j+t-1}^{i+s}\right)$ contributes $\tau_{i+s, j+t-1} \psi_{k-1, s, t-1}$.

So we obtain 


$$
\begin{aligned}
\psi_{k, s, t}= & \left(\prod_{\ell=0}^{s-1} \theta_{i+\ell, j+\ell+t-s}\right)\left(\prod_{\ell=0}^{t-s-1} \tau_{i, j+\ell}\right) \\
& \times\left[\eta_{j+t} h_{k-1-t}\left(\eta_{j}, \ldots, \eta_{j+t}\right) h_{s}\left(1, \zeta, \ldots, \zeta^{t-s}\right)\right. \\
& \left.\quad+h_{k-t}\left(\eta_{j}, \ldots, \eta_{j+t-1}\right) h_{s-1}\left(1, \zeta, \ldots, \zeta^{t-s}\right)\right]
\end{aligned}
$$

To simplify the last summand, we use (9.5) to compute

$$
\begin{aligned}
\tau_{i+s, j+t-1} \prod_{\ell=0}^{s-1} \theta_{i+\ell, j+\ell+t-1-s} \\
=\left(\tau_{i+s, j+t-1}\right) \theta_{i+s-1, j+t-2} \theta_{i+s-2, j+t-3} \cdots \theta_{i, j+t-1-s} \\
=\zeta \theta_{i+s-1, j+t-1}\left(\tau_{i+s-1, j+t-2}\right) \theta_{i+s-2, j+t-3} \cdots \theta_{i, j+t-1-s} \\
=\zeta^{2} \theta_{i+s-1, j+t-1} \theta_{i+s-2, j+t-2}\left(\tau_{i+s-2, j+t-3}\right) \cdots \theta_{i, j+t-1-s} \\
\quad \cdots \\
=\zeta^{s}\left(\tau_{i, j+t-s-1}\right) \prod_{\ell=0}^{s-1} \theta_{i+\ell, j+\ell+t-s} .
\end{aligned}
$$

By (9.7) and (9.8), we now have

$$
\begin{aligned}
\psi_{k, s, t}=\left(\prod_{\ell=0}^{s-1} \theta_{i+\ell, j+\ell+t-s}\right) & \left(\prod_{\ell=0}^{t-s-1} \tau_{i, j+\ell}\right) \\
\times & \eta_{j+t} h_{k-1-t}\left(\eta_{j}, \ldots, \eta_{j+t}\right) h_{s}\left(1, \zeta, \ldots, \zeta^{t-s}\right) \\
& +h_{k-t}\left(\eta_{j}, \ldots, \eta_{j+t-1}\right) h_{s-1}\left(1, \zeta, \ldots, \zeta^{t-s}\right) \\
& \left.+h_{k-t}\left(\eta_{j}, \ldots, \eta_{j+t-1}\right) \zeta^{s} h_{s}\left(1, \zeta, \ldots, \zeta^{t-s-1}\right)\right] .
\end{aligned}
$$

The factor of $\zeta^{s}$ in the last term can be absorbed into the (homogeneous) polynomial $h_{s}$ to make the sum of the last two terms equal to

$$
h_{k-t}\left(\eta_{j}, \ldots, \eta_{j+t-1}\right)\left(h_{s-1}\left(1, \zeta, \ldots, \zeta^{t-s}\right)+h_{s}\left(\zeta, \zeta^{2}, \ldots, \zeta^{t-s}\right)\right) .
$$

Now, taking $x_{t-s-i}=\zeta^{i}$, (9.2) implies that the two terms in the parenthesis simplify to $h_{s}\left(1, \zeta, \ldots, \zeta^{t-s}\right)$. Thus, (9.9) yields

$$
\begin{aligned}
\psi_{k, s, t}=\left(\prod_{\ell=0}^{s-1} \theta_{i+\ell, j+\ell+t-s}\right) & \left(\prod_{\ell=0}^{t-s-1} \tau_{i, j+\ell}\right) h_{s}\left(1, \zeta, \ldots, \zeta^{t-s}\right) \\
& \times\left[\eta_{j+t} h_{k-1-t}\left(\eta_{j}, \ldots, \eta_{j+t}\right)+h_{k-t}\left(\eta_{j}, \ldots, \eta_{j+t-1}\right)\right] .
\end{aligned}
$$


Again applying (9.2), we find that (9.6) holds, and the induction is complete.

We can apply this result to show that $x^{n}$ acts by 0 in the following cases.

Lemma 9.10. In the notation of Proposition 3.5, we have $x^{n} \cdot e_{i}=0$.

Proof. Fix $i$. We will apply Lemma 9.4 in the case $k=n, m=m^{\prime}$, with $X=x$ and $v_{i}^{i}=e_{i}$ for $1 \leq i \leq m$. The $v_{j}^{i}$ do not play a role in the proof for $i \neq j$, so we set $\theta_{i, j}=\tau_{i, j}=0$ when $i \neq j$ and assume $i=j$ for the remainder of the proof. From the proof of Proposition 3.5, we have $\eta_{i}=\gamma \zeta^{i}, \theta_{i, i}=-\gamma \zeta^{i+1}$, and $\tau_{i, i}=0$ for all $i$. So, (9.5) holds and we can apply Lemma 9.4. Making these substitutions into (9.6), we immediately see that $\psi_{n, s, t}=0$ unless $s=t$, by considering the $\tau$ factor. In case $s=t$, the $\tau$ factor and the last factor are equal to 1 . This gives

$$
\psi_{n, s, s}=\left(\prod_{\ell=0}^{s-1} \theta_{i+\ell, i+\ell}\right) h_{n-s}\left(\eta_{i}, \eta_{i+1}, \cdots, \eta_{i+s}\right) .
$$

Since $h_{n-s}\left(\eta_{i}, \eta_{i+1}, \cdots, \eta_{i+s}\right)$ is a scalar multiple of $h_{n-s}\left(1, \zeta, \ldots, \zeta^{s}\right)$, this vanishes by Lemma 9.3 unless $s=0$ or $s=n$.

So we have $x^{n} \cdot e_{i}=\left(\psi_{n, 0,0}+\psi_{n, n, n}\right) e_{i}$. These are easily computed by substitution to be

$$
\begin{gathered}
\psi_{n, 0,0}=h_{n}\left(\eta_{i}\right)=\left(\eta_{i}\right)^{n}=\gamma^{n} \\
\psi_{n, n, n}=\prod_{\ell=0}^{n-1} \theta_{i+\ell, i+\ell}=(-1)^{n} \gamma^{n} \prod_{\ell=0}^{n-1} \zeta^{i+\ell+1}=(-1)^{n} \gamma^{n} \prod_{\ell=0}^{n-1} \zeta^{\ell}=-\gamma^{n},
\end{gathered}
$$

where the last equality invokes Equation (9.1). Thus we have shown that $x^{n} \cdot e_{i}=0$.

Lemma 9.11. In the notation of Theorem 6.1, we have $x^{n} \cdot a_{j}^{i}=0$ for all $(i, j)$.

Proof. We will apply Lemma 9.4 in the case $k=n$ with $X=x$ and $v_{j}^{i}=a_{j}^{i}$. From the proof of Theorem 6.1, we have $\eta_{j}=\gamma \zeta^{j}, \theta_{i, j}=-\gamma \mu_{i, j} \zeta^{i+1}$, and $\tau_{i, j}=\lambda_{i, j}$, and that these satisfy (9.5). Now making these substitutions into (9.6), we see that $\psi_{n, s, t}$ is a scalar times

$$
h_{s}\left(1, \zeta, \ldots, \zeta^{t-s}\right) h_{n-t}\left(1, \zeta, \ldots, \zeta^{t}\right)
$$

By Lemma 9.3, the second factor vanishes except when $t=0$ or $t=n$. Then, again by Lemma 9.3, the first factor vanishes except when $s=0$ or $s=t=n$. Therefore, we need only consider the cases when $(s, t)$ equals $(0,0),(0, n)$, and $(n, n)$, in which these factors both equal 1 . Notice that we have in all of these cases, $a_{j+t}^{i+s}=a_{j}^{i}$, because the indices for arrows are taken modulo $n$. So it suffices to show 
that $\psi_{n, 0,0}+\psi_{n, 0, n}+\psi_{n, n, n}=0$. Substitute and compute, omitting factors equal to 1 . We get

$$
\psi_{n, 0,0}=h_{n}\left(\eta_{j}\right)=\left(\eta_{j}\right)^{n}=\gamma^{n} \quad \text { and } \quad \psi_{n, 0, n}=\prod_{\ell=0}^{n-1} \tau_{i, j+\ell}=0
$$

The latter follows by the second condition of Theorem 6.1 , because there will be a factor with $i=j+\ell$ modulo $n$. Further,

$$
\psi_{n, n, n}=\prod_{\ell=0}^{n-1} \theta_{i+\ell, j+\ell}=(-1)^{n} \gamma^{n} \prod_{\ell=0}^{n-1} \mu_{i+\ell, j+\ell} \zeta^{i+\ell+1}=(-1)^{n} \gamma^{n} \prod_{\ell=1}^{n} \zeta^{\ell}=-\gamma^{n}
$$

where the penultimate equality uses (4.2) and the last equality invokes Equation (9.1). Thus we have shown that $x^{n} \cdot a_{j}^{i}=0$.

Lemma 9.12. In the notation of Theorem 6.3 , we have $x^{n} \cdot b_{j}^{i}=0$ for all $(i, j)$.

Proof. Again, we apply Lemma 9.4 with $k=n, X=x$, and $v_{j}^{i}=b_{j}^{i}$. The proof is the same as for Lemma 9.11, except in this case we find that

$$
\psi_{n, 0,0}=\gamma_{-}^{n}, \quad \psi_{n, n, n}=-\gamma_{+}^{n}, \quad \text { and } \quad \psi_{n, 0, n}=\prod_{\ell=0}^{n-1} \tau_{i, j+\ell}=\prod_{\ell=0}^{n-1} \lambda_{i, j+\ell}
$$

Thus, we see that $x^{n}$ acts by 0 if and only if $\left(\gamma_{+}\right)^{n}=\left(\gamma_{-}\right)^{n}+\prod_{\ell=0}^{n-1} \lambda_{i, j+\ell}$, which is the condition assumed on the scalars in Theorem 6.3.

\section{Acknowledgments}

We thank Steven Sam for directing us to the reference for principal specializations of symmetric polynomials. We also thank Mio Iovanov for helping us with the proof of Lemma 2.5, and Susan Montgomery for suggesting the extensions of our results in Section 8. We are grateful to the referee for careful comments and suggestions. Walton was supported by the National Science Foundation: NSF-grant DMS-1550306.

\section{References}

[Assem et al. 2006] I. Assem, D. Simson, and A. Skowroński, Elements of the representation theory of associative algebras, I: Techniques of representation theory, London Mathematical Society Student Texts 65, Cambridge University Press, Cambridge, 2006. MR 2006j:16020 Zbl 1092.16001

[Banica 2005] T. Banica, "Quantum automorphism groups of homogeneous graphs", J. Funct. Anal. 224:2 (2005), 243-280. MR 2006d:16061 Zbl 1088.46040

[Banica et al. 2007] T. Banica, J. Bichon, and G. Chenevier, "Graphs having no quantum symmetry", Ann. Inst. Fourier (Grenoble) 57:3 (2007), 955-971. MR 2008f:20004 Zbl 1178.05047 
[Bates et al. 2012] T. Bates, D. Pask, and P. Willis, "Group actions on labeled graphs and their $C^{*}$-algebras”, Illinois J. Math. 56:4 (2012), 1149-1168. MR 3231477 Zbl 1292.46034

[Bichon 2003] J. Bichon, "Quantum automorphism groups of finite graphs", Proc. Amer. Math. Soc. 131:3 (2003), 665-673. MR 2003j:16049 Zbl 1013.16032

[Chen 1999] H.-X. Chen, "A class of noncommutative and noncocommutative Hopf algebras: the quantum version”, Comm. Algebra 27:10 (1999), 5011-5032. MR 2000g:16044 Zbl 0942.16038

[Chen et al. 2004] X.-W. Chen, H.-L. Huang, Y. Ye, and P. Zhang, "Monomial Hopf algebras", J. Algebra 275:1 (2004), 212-232. MR 2005e:16060 Zbl 1071.16030

[Cibils and Rosso 1997] C. Cibils and M. Rosso, "Algèbres des chemins quantiques", Adv. Math. 125:2 (1997), 171-199. MR 98c:16048 Zbl 0867.17012

[Cibils and Rosso 2002] C. Cibils and M. Rosso, "Hopf quivers", J. Algebra 254:2 (2002), 241-251. MR 2003h:16016 Zbl 1020.16025

[Gordienko 2015] A. S. Gordienko, "Algebras simple with respect to a Taft algebra action", J. Pure Appl. Algebra 219:8 (2015), 3279-3291. MR 3320219 Zbl 1323.16020

[Huang and Liu 2010] H.-L. Huang and G. Liu, "On quiver-theoretic description for quasitriangularity of Hopf algebras”, J. Algebra 323:10 (2010), 2848-2863. MR 2011d:16047 Zbl 1230.16028

[Huang et al. 2010] H.-L. Huang, Y. Ye, and Q. Zhao, "Hopf structures on the Hopf quiver $Q(\langle g\rangle, g)$ ", Pacific J. Math. 248:2 (2010), 317-334. MR 2011m:16068 Zbl 1262.16029

[Kumjian and Pask 1999] A. Kumjian and D. Pask, " $C^{*}$-algebras of directed graphs and group actions”, Ergodic Theory Dynam. Systems 19:6 (1999), 1503-1519. MR 2000m:46125 Zbl 0949.46034

[Majid 1991] S. Majid, "Doubles of quasitriangular Hopf algebras", Comm. Algebra 19:11 (1991), 3061-3073. MR 92k:16052 Zbl 0767.16014

[Majid 1994] S. Majid, “Cross products by braided groups and bosonization”, J. Algebra 163:1 (1994), 165-190. MR 94m:18009 Zbl 0807.16036

[Montgomery 1993] S. Montgomery, Hopf algebras and their actions on rings, CBMS Regional Conference Series in Mathematics 82, Providence, RI, 1993. MR 94i:16019 Zbl 0793.16029

[Montgomery and Schneider 2001] S. Montgomery and H.-J. Schneider, "Skew derivations of finitedimensional algebras and actions of the double of the Taft Hopf algebra", Tsukuba J. Math. 25:2 (2001), 337-358. MR 2002h:16055 Zbl 1032.16031

[van Oystaeyen and Zhang 2004] F. van Oystaeyen and P. Zhang, "Quiver Hopf algebras", J. Algebra 280:2 (2004), 577-589. MR 2005f:16068 Zbl 1079.16029

[Radford 2012] D. E. Radford, Hopf algebras, Series on Knots and Everything 49, World Scientific Publishing, Hackensack, NJ, 2012. MR 2894855 Zbl 1266.16036

[Schiffler 2014] R. Schiffler, Quiver representations, Springer, Cham, Switzerland, 2014. MR 3308668 Zbl 1310.16015

[Stanley 1999] R. P. Stanley, Enumerative combinatorics, vol. 2, Cambridge Studies in Advanced Mathematics 62, Cambridge University Press, Cambridge, 1999. MR 2000k:05026 Zbl 0945.05006 [Zhang 2006] P. Zhang, "Hopf algebras on Schurian quivers", Comm. Algebra 34:11 (2006), 40654082. MR 2007m:16056 Zbl 1168.16023

Communicated by Susan Montgomery

Received 2015-01-06 Revised 2015-07-30 Accepted 2015-09-19

ryan-kinser@uiowa.edu

Department of Mathematics, University of lowa, lowa City, IA 52242, United States

notlaw@temple.edu

Department of Mathematics, Temple University, Philadelphia, PA 19122, United States 


\section{Algebra \& Number Theory}

msp.org/ant

\section{EDITORS}

MANAGING EDITOR

Bjorn Poonen

Massachusetts Institute of Technology

Cambridge, USA

\author{
EDITORIAL BOARD CHAIR \\ David Eisenbud \\ University of California \\ Berkeley, USA
}

BOARD OF EDITORS

Georgia Benkart

Dave Benson

Richard E. Borcherds

John H. Coates

J-L. Colliot-Thélène

Brian D. Conrad

Hélène Esnault

Hubert Flenner

Sergey Fomin

Edward Frenkel

Andrew Granville

Joseph Gubeladze

Roger Heath-Brown

Craig Huneke

Kiran S. Kedlaya

János Kollár

Yuri Manin

Philippe Michel
University of Wisconsin, Madison, USA

University of Aberdeen, Scotland

University of California, Berkeley, USA

University of Cambridge, UK

CNRS, Université Paris-Sud, France

Stanford University, USA

Freie Universität Berlin, Germany

Ruhr-Universität, Germany

University of Michigan, USA

University of California, Berkeley, USA

Université de Montréal, Canada

San Francisco State University, USA

Oxford University, UK

University of Virginia, USA

Univ. of California, San Diego, USA

Princeton University, USA

Northwestern University, USA

École Polytechnique Fédérale de Lausanne
Susan Montgomery

Shigefumi Mori

Raman Parimala

Jonathan Pila

Anand Pillay

Victor Reiner

Peter Sarnak

Joseph H. Silverman

Michael Singer

Vasudevan Srinivas

J. Toby Stafford

Ravi Vakil

Michel van den Bergh

Marie-France Vignéras

Kei-Ichi Watanabe

Efim Zelmanov

Shou-Wu Zhang
University of Southern California, USA

RIMS, Kyoto University, Japan

Emory University, USA

University of Oxford, UK

University of Notre Dame, USA

University of Minnesota, USA

Princeton University, USA

Brown University, USA

North Carolina State University, USA

Tata Inst. of Fund. Research, India

University of Michigan, USA

Stanford University, USA

Hasselt University, Belgium

Université Paris VII, France

Nihon University, Japan

University of California, San Diego, USA

Princeton University, USA

\section{PRODUCTION}

production@msp.org

Silvio Levy, Scientific Editor

See inside back cover or msp.org/ant for submission instructions.

The subscription price for 2016 is US $\$ 290 /$ year for the electronic version, and $\$ 485 /$ year ( $+\$ 55$, if shipping outside the US) for print and electronic. Subscriptions, requests for back issues and changes of subscribers address should be sent to MSP.

Algebra \& Number Theory (ISSN 1944-7833 electronic, 1937-0652 printed) at Mathematical Sciences Publishers, 798 Evans Hall \#3840, c/o University of California, Berkeley, CA 94720-3840 is published continuously online. Periodical rate postage paid at Berkeley, CA 94704, and additional mailing offices.

ANT peer review and production are managed by EditFLOW ${ }^{\circledR}$ from MSP.

\section{PUBLISHED BY}

- mathematical sciences publishers

nonprofit scientific publishing

http://msp.org/

(C) 2016 Mathematical Sciences Publishers 


\section{Algebra \& Number Theory}

Volume $10 \quad$ No. $1 \quad 2016$

Stable sets of primes in number fields

ALEXANDER IVANOV

Hopf-Galois structures arising from groups with unique subgroup of order $p$

Tiмотну KонL

On tensor factorizations of Hopf algebras

Marc Keilberg and Peter Schauenburg

Extension theorems for reductive group schemes

ADRIAN VASIU

Actions of some pointed Hopf algebras on path algebras of quivers

RYAN Kinser and CHELSEA WALTON

On the image of the Galois representation associated to a non-CM Hida family

JACLYN LANG

Linear relations in families of powers of elliptic curves

FABRIZIO BARROERO and LAURA CAPUANO 